\title{
Quantum Dot bioconjugates for diagnostic applications
}

Maria Diaz Gonzalez ${ }^{(1)}$, Alfredo de la Escosura ${ }^{(1)}$, Maria Teresa Fernandez-Argüelles ${ }^{(1)}$, Francisco Javier Garcia Alonso ${ }^{(2)}$ and Jose Manuel Costa-Fernandez ${ }^{(1)(*)}$

(1) University of Oviedo. Department of Physical and Analytical Chemistry. Avda. Julian Claveria, 8. 33006 Oviedo. Spain.

(2) University of Oviedo. Department of Organic and Inorganic Chemistry. Avda. Julian Claveria, 8. 33006 Oviedo. Spain.

${ }^{(*)}$ Corresponding author.

Email: jcostafe@uniovi.es

ORCID: 0000-0002-8671-5300 


\begin{abstract}
Quantum Dots (QDs) are a special type of engineered nanomaterials with outstanding optoelectronic properties make them as a very promising alternative to conventional luminescent dyes for biomedical applications, including biomolecule targeting, luminescence imaging as well as in drug delivery.

A key parameter to ensure successful biomedical applications of QDs is that the surface of these nanomaterials should be modified with appropriate functional groups to ensure stability in aqueous solutions and conjugated with recognition elements capable to ensure an efficient tagging of the biomolecules of interest.

Here, we present a review summarizing most relevant strategies for QDs surface modification and for their conjugation to biomolecules for preparation of nanoplatforms for luminescent biomolecule sensing and imaging-guided targeting. Applications of conjugations of photoluminescent QDs with different biomolecules in both in vitro and in vivo chemical sensing, immunoassays or luminescence imaging are revised. Recent progress in application of functionalized QDs in ultrasensitive detection in bioanalysis, diagnostics and imaging strategies developments are here included. Finally, some key future research goals in the progress of bioconjugation of QDs for diagnosis are identified, including novel synthetic approaches, the need of exhaustive characterization of bioconjugates or the design of signal amplification schemes.
\end{abstract}

Keywords: nanoparticles; quantum dots; luminescence; surface functionalization; diagnostics; biosensing. 


\section{INTRODUCTION}

Nanotechnology comprises the fabrication, characterization, manipulation and application of materials that present at least one dimension within 1-100 nm. When the size of the material is reduced below such threshold, they behave differently from the same material with macroscopic dimensions, giving rise to unique and extraordinary physicochemical properties due to the quantum confinement of the electrons when the dimensions are smaller than the Bohr radius [1].

Nanomaterials can be classified according to different criteria, being the most frequent that based on the dimensions of the nanomaterials. Three-dimensional (3-D) nanomaterials are those whose three dimensions are higher that the nanometer scale, but are composed of individual building blocks that are in the nanometer scale, such as nanocomposites. Twodimensional (2-D) nanomaterials are those that present one dimension in the nanometer scale, including thin films or nanocoatings. One-dimensional (1-D) nanomaterials possess two dimensions within 1-100 nm and only one dimension bigger than the nanometer scale, such as such as nanotubes, nanorods, nanowires, etc. And finally, Zero-dimensional (0-D) nanomaterials are those where the three dimensions are within 1-100 nm, where nanoparticles (NPs) are included, such as metal NPs, semiconductor quantum dots (QDs), or carbon based quantum dots (C-dots) [2].

In the last decades the development of novel nanomaterials have gained increasing interest due to their fascinating physicochemical properties, which offer a great potential in different fields, such as (bio)analytical chemistry [3,4], water treatment [5], catalysis, electrocatalysis [6-9], cancer treatment [10], energy storage devices [11], etc. Although there is no much information available about production of QDs, it was possible to estimate worldwide and Europe-wide production and use of ten different nanomaterials, including quantum dots, from a survey sent to companies producing and using engineered nanomaterials. From such studies, production of QDs was estimated to be of about 10 t/year or lower [12].

Inorganic semiconductor nanocrystals, or QDs-have demonstrated a range of unique optoelectronic properties and can offer new attractive options in many biomedical applications [13-16]. For example, QDs have been widely employed for fluorescence sensing 
and bioimaging due to their exceptional photoluminescent characteristics including the capabilities of tune their emission wavelengths just by controlling the NP size. Although conventional organic fluorescent molecules are widely used for bioimaging applications, QDs are superior luminescence tags in terms of their photophysical properties: QDs have broad excitation spectra with a narrow and sharp emission spectra and large Stocks shifts $(>100 \mathrm{~nm})$. Such optoelectronic properties are of great value for multiplexed applications $[17,18]$ as using a single light source it is possible to simultaneously excite multiple QDs of different sizes (multiple emission peaks). Additionally, QDs exhibit very high molar adsorption coefficients and higher quantum yields as compared with organic fluorophores. As a consequence, such fluorescent NPs are nearly 20 times brighter and thousands of times more stable against photobleaching than conventional organic dyes $[19,20]$. Such exceptional optical behavior justify why QDs have promptly emerged as valuable photoluminescent probes in many analytical applications.

Particularly, the use of NPs for diagnostic applications is exponentially increasing due to their highly valuable optoelectronic properties and small size. Additionally, advances performed in the last years on surface modification and functionalization have given rise to improved colloidal stability in complex media and biological buffers and biocompatibility, while allowing their bonding to recognition elements [21]. In this sense, it is especially relevant the development carried out in the field of synthesis of NPs with interesting optical properties that overcome limitations of traditional organic dyes, including gold nanoparticles (AuNPs), semiconductor QDs gold nanoclusters, silver nanoclusters, rare-earth based NPs, carbon dots or dye-loaded NPs [22].

However, despite all the exceptional properties demonstrated by QDs to date, their applications in clinical analysis, especially during the first decades of development, can be considered still somehow limited, in part due to their low targeting efficiency and eventual high toxicity that could hinder their application for in vivo imaging. This explains that many research efforts are being pursued in the development of QDs exhibiting low biological toxicity (e.g. those based on an $\mathrm{Ag}_{2} \mathrm{~S}$ core) [23]. Additionally, in absence of any molecular moiety being attached to the NP surface, QDs generally show nonselective distribution across the different organisms, thus failing to satisfy the minimum requirements for appropriate molecular imaging. Clearly, QDs-based nanoprobes development require a previous surface 
functionalization of the NPs to aid various approaches of targeting-guided imaging techniques. In addition, to ensure a needed biocompatibility of the nanoprobes to be used in in vivo imaging or sensing, a proper nanoparticle-surface functionalization is required.

This review intends to highlight advances carried out in the use of QDs for diagnostic applications. Following an introduction that covers a brief description of the main types of quantum dots used in bioanalysis the article deals with the strategies of solubilization and stabilization of QDs in aqueous solutions under physiological conditions. Next, approaches for functionalization of QDs with biological molecules are summarized, a key aspect required before they can be employed for the detection of analytes in biological matrices. Next part describes some of the most relevant applications of QD bioconjugates in optical imaging of biomarkers. In vitro applications of QDs in medicine, where different detection schemes based on the bioconjugation of QDs to antibodies, aptamers, peptides or other types of recognition elements are also reviewed. In this context, an overview of recent advances in the development of low-cost, portable and easy to use QD-based biosensing devices for clinical applications (point-of-care) is given. A final section of future prospects intends to provide which are the next steps where the research on the use of QDs in diagnostic applications is heading to. 


\section{QUANTUM DOTS: NATURE AND TYPES.}

Despite many initial problems of lack of reproducibility and reduced optical quality related to the synthesis of colloidal fluorescent semiconductor NPs, important advances have been achieved in this field. In pioneer studies on the routine preparation of colloidal QDs, the QDs core was usually capped with an organic layer, that coordinates to core-metal sites and stabilizes QDs' surface preventing an irreversible flocculation (aggregation) of the nanocrystals [24]. Unfortunately, these protective ligands are hydrophobic and thus nanocrystals capped with those coatings are not compatible with bioanalytical assay conditions. Consequently, QDs surface should be further modified by appropriate hydrophilic functional groups to allow their dispersion in aqueous solutions while maintaining their high photoluminescence quantum yield.

Although fluorescence emission is the most exploited property of QDs, doping the core of the nanocrystals with transition elements has been exploited to provide the QDs with new improved multimodal characteristics for biomedical applications. Additionally, during recent years, many research groups have tried to overcome the problem of eventual cytotoxicity of more conventional heavy-metal based QDs. Approaches based on carbon-based nanomaterials are one of the most promising strategies. In this section, a brief revision of the nature and characteristics of QDs typically employed in clinical and biomedical applications is presented.

\subsection{Semiconductor QDs}

QDs are spherical semiconductor photoluminescent NPs with a diameter between 2-10 $\mathrm{nm}$. Since the dimensions of the NP are smaller than the Bohr radius, the energy levels are quantized. As consequence, the optoelectronic properties of QDs depend on their size due to quantum confinement effects, and differ from the properties observed for the same bulk material [25]. In fact, due to the quantized energy levels, QDs present an intense emission of photoluminescence: when the semiconductor QD is irradiated with a light source, the absorption produces a movement of an electron from the lower energy valence band to a higher energy conduction band. Then, an electron-hole pair is generated, and its 
recombination gives rise to the emission of intense photoluminescence. Additionally, other optical features that make QDs very appealing for analytical applications include broad absorption spectra, narrow and symmetric emission bands that can be tuned by changing the composition and size of the NP (see Figure 1), large Stoke shifts, and high photostability $[26,27]$.

Since the energy band gap decreases as the QD diameter increases, the emission wavelength is shifted to longer wavelengths. Hence, for the same composition of the QD, the emission can be tuned by just modifying the size of the NP (size-dependent emission). In addition, the QD can be synthesized with different semiconductors, e.g. CdS, CdSe, CdTe, $\mathrm{ZnS}, \mathrm{ZnSe}, \mathrm{PbS}$, InP, etc, that also affect to their spectral properties. Those features are very attractive, because emission in a wide range of the spectra, from the UV up to the NIR, can be obtained by changing the composition and size of the QD. Additionally, they all present broad absorption bands, allowing the use of a single excitation source to efficiently excite QDs with different emission wavelengths, which, besides their high luminescent quantum yields and long photostability (e.g.: CdS/ZnS QDs are nearly 20 times brighter and 100 times more stable than the widely used Rhodamine 6G) [19], is of high interest to perform multianalyte detection $[16,28]$.

The synthesis of semiconductor QDs can be performed in both, organic hydrophobic solvent and aqueous media. Nevertheless, the synthetic routes that generate QDs with the best optoelectronic properties are those carried out in non-polar solvents and using hydrophobic ligands. As consequence, the QDs obtained through these routes present outstanding photoluminescent properties, but they tend to aggregate and precipitate in aqueous solutions. Hence, it is necessary to perform a surface modification of the QDs in order to protect their surface with molecules that present hydrophilic groups oriented towards the medium, rendering good colloidal stability to the QDs in aqueous media. The most common strategies to transfer QDs from hydrophobic organic media to aqueous solution are summarized in the next QDs surface functionalization section. 


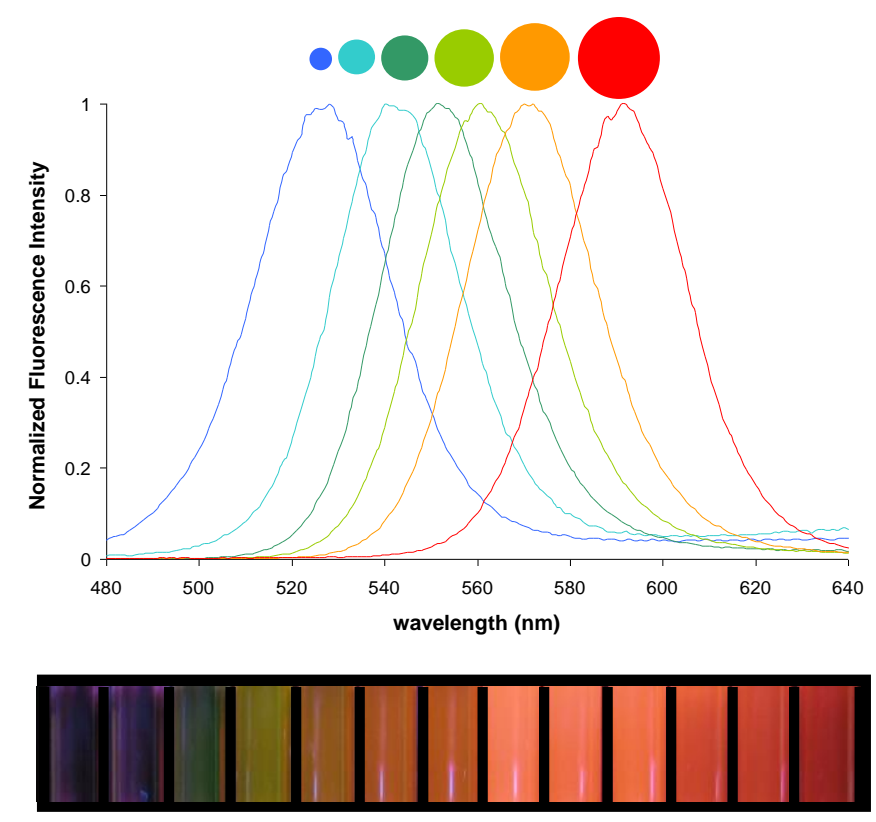

Figure 1. Size-tuneable fluorescence spectra of CdSe QDs with different diameter sizes. At the bottom images of the colloidal suspensions of the different sized QDs under UV light [29]. Reprinted from Fernandez-Argüelles MT, Costa-Fernandez JM, Pereiro R, Sanz-Medel A, Organically Modified Quantum Dots in Chemical and Biochemical Analysis. In: Rurack K, Martinez-Manez R (Ed) The Supramolecular Chemistry of Organic-Inorganic Hybrid Materials, Copyright (2010), with permission from Wiley.

\subsection{Metal-doped QDs.}

In the last years, advances carried out in the rational design of nanomaterials have contributed to the development of hybrid NPs that combine the interesting size- and shapedependent properties of semiconductor QDs with a long-lived phosphorescence-type emission. In this sense, incorporation of suitable atoms or ions into host lattices have generated a novel type of QDs with that are very promising in bioanalytical applications [30]. $\mathrm{ZnS}, \mathrm{ZnO}, \mathrm{ZnSe}, \mathrm{CdS}$ and $\mathrm{CdSe}$ QDs can be used as host lattices to incorporate other transition-metal and lanthanide ions, including $\mathrm{Mn}^{2+}, \mathrm{Cu}^{2+}, \mathrm{Co}^{2+}, \mathrm{Ni}^{2+}, \mathrm{Ag}^{+}, \mathrm{Pb}^{2+}, \mathrm{Cr}^{3+}, \mathrm{Eu}^{3+}$, $\mathrm{Tb}^{3+}, \mathrm{Sm}^{3+}$ and $\mathrm{Er}^{3+}$ as dopant agents, giving rise to luminescent QDs with novel properties [31]. In this sense, host lattices as $\mathrm{ZnS}$ and $\mathrm{ZnO}$ are being widely studied because they do not contain toxic metals, thus presenting potentially lower toxicity, and also because they are characterized by a larger energy band gap, which permits the incorporation of more doping agents, which is very appealing for the development of dual-doped QDs [32,33]. Introduction 
of the dopant typically increases the photoluminescence lifetime of the QD (see Figure 2), producing a phosphorescence-like emission that overcomes the limitations of fluorescent NPs or dyes due to the removal of the fluorescence background commonly found in biosensing and bioimaging applications [14].
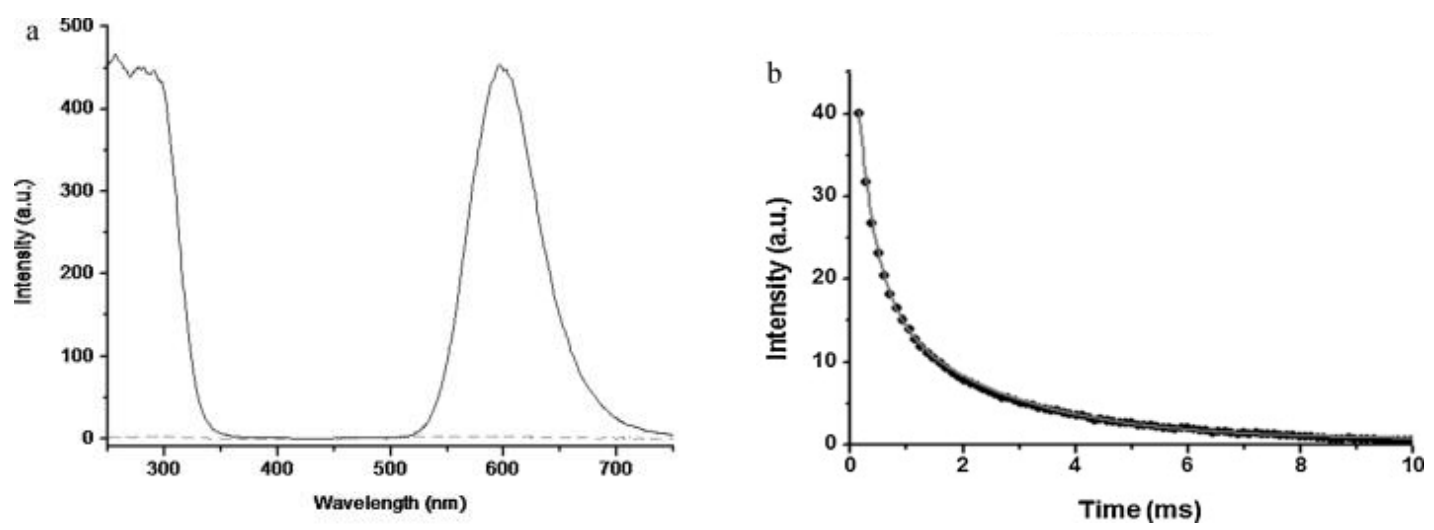

Figure 2. (a) Excitation and emission spectra of colloidal Mn:ZnS QDs (solid-line) and of colloidal ZnS QDs (dotted line) and (b) Decay curve of luminescence emission of colloidal Mn:ZnS QDs with a first lifetime component in the range of $0.3 \mathrm{~ms}$ and a second longer lifetime component around 2.1 ms [34]. Reprinted from Publication Anal Chim Acta 712, Sotelo-Gonzalez E, Fernandez-Arguelles MT, Costa-Fernandez JM, Sanz-Medel A, Mn-doped ZnS quantum dots for the determination of acetone by phosphorescence attenuation, 120-126, Copyright (2012), with permission from Elsevier.

Most of the synthetic routes described to incorporate the doping agent into the host structure are based in wet chemistry procedures carried out in organic media typically under high temperatures, or in aqueous media through precipitation or microemulsion methods in order to control the size and shape of the nanocrystal as well as a homogeneous distribution of the dopant in the host matrix.

\subsection{QDs based on Carbon.}

Despite inorganic semiconductor QDs have been deeply evaluated as luminescent nanomaterials for bioanalytical applications, carbon-based NPs such as carbon quantum dots (C-dots) and graphene quantum dots (GQDs) have become an attractive alternative due to their high photoluminescence quantum yields, low photobleaching effects, high biocompatibility and low toxicity, while avoiding the use of heavy metals commonly found 
in semiconductor QDs [35]. Additionally, they present an exceptional colloidal stability in aqueous media as consequence of their small size, since the Brownian motion provides sufficient energy to inhibit aggregation between them [36,37].

Nevertheless, probably the most remarkable property of C-dots is their excitation wavelength-dependent fluorescence emission (see Figure 3), which makes them an excellent alternative for optical imaging applications [38]. The principle of such characteristic emission is not fully understood, and nowadays there is still controversy about the origin of the fluorescence emission of C-dots. In this sense, C-dots obtained through different synthetic routes or using different precursors present different optical behaviour, suggesting that carbon based QDs are quite complex. In fact, there is no agreement within the scientific community to explain the optical properties of $\mathrm{C}$-dots, that have been attributed to surface state emission, intrinsic band emission, triple ground state emission, dipole emission involving electron-phonon coupling, transition from surface electrons to valence holes, selftrapped excitons and even to the presence of small organic molecules $[39,40]$.

Typically, synthesis of C-dots has employed graphite as carbon source, and a surface passivation of the $\mathrm{C}$-dots is frequently necessary in order to obtain better fluorescent properties. However, green methods for synthesis of C-dots based on the use of natural precursors are gaining importance, since besides the cost-effective and environmental friendly syntheses, the C-dots obtained do not need any surface modification, and present high photoluminescence as well as excellent stability in aqueous media [41].

Recently, capabilities of C-dots for diagnostic applications were expanded by metal doping of the NPs with $\mathrm{N}$ and lanthanides (e.g. Gd and $\mathrm{Yb}$ ) resulting in co-doped nanomaterials exhibiting not only strong fluorescence but also high Magnetic Resonance and Computed Tomography contrast capabilities [42]. Such simple approach allowed to prepare multimodal QDs for bioimaging applications. Still some work in this area is needed to study of the surface modification of these co-doped NPs with appropriate recognition biomolecules for targeted bioimaging. 

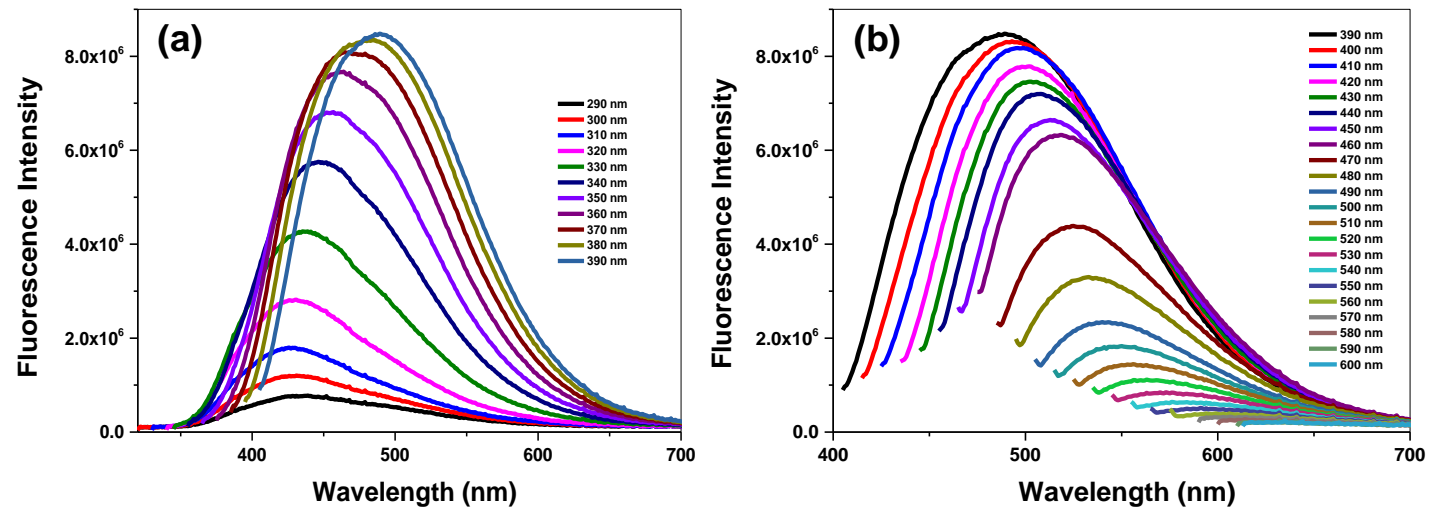

Figure 3. Emission spectra of black pepper C-dots under different excitation wavelengths (a) from 290 to $390 \mathrm{~nm}$ and (b) from 390 to $600 \mathrm{~nm}$. Reprinted with permission from [39]. Copyright 2018 Beilstein-Institut. Reprinted from Vasimalai N, Vilas-Boas V, Gallo J, Cerqueira MF, MenéndezMiranda M, Costa-Fernández JM, Diéguez L, Espiña B, Fernández-Argüelles MT, Beilstein J Nanotechnol 2018, 9, 530-544.

\section{QUANTUM DOT AQUEOUS STABILIZATION STRATEGIES}

As mentioned before, conventional high-quality fluorescent QDs are commonly synthesized in organic solvents at high temperature. However, the QDs should be made water-compatible (stable in aqueous and biological media maintaining their optoelectronic properties) if intended to be used in bioanalytical applications. For such purpose, surface modification of the QDs after synthesis is a must. Furthermore, QDs should have functional groups available for further bioconjugation to biomolecules on their surface.

An appropriate QDs surface passivation also can solve some of the problems typically affecting these NPs: first, crystalline NPs can easily form surface defects that quench the fluorescence properties of naked QDs [43]. In addition, naked QDs can suffer from surface oxidation, photochemical degradation and/or leaching of metal ions from the NP core after exposure of QDs to ionic or biological media affecting to their optoelectronic properties and producing undesirable cytotoxicity [44]. Thus, modification of the QDs surface with appropriate ligands is essential not only to stabilize the NPs in physiological media (particularly important if they are going to be applied to clinical uses) but also to reduce 
nanocrystal surface defects, minimizing the QDs reactivity and toxicity. Moreover, despite the significant progress achieved in the synthesis of QDs, biological uses of QDs require to render such NPs into biocompatible probes. In this context, the availability of robust and versatile NP surface chemistries are invaluable strategies to achieve QDs stabilization in biological buffers, keeping their original photophysical properties, and providing adequate reactive groups for further bioconjugations. There are three main strategies employed for hydrophilization of QDs (based on the attachment of polar functional groups to the surface of the QD), which are summarized in Figure 4.

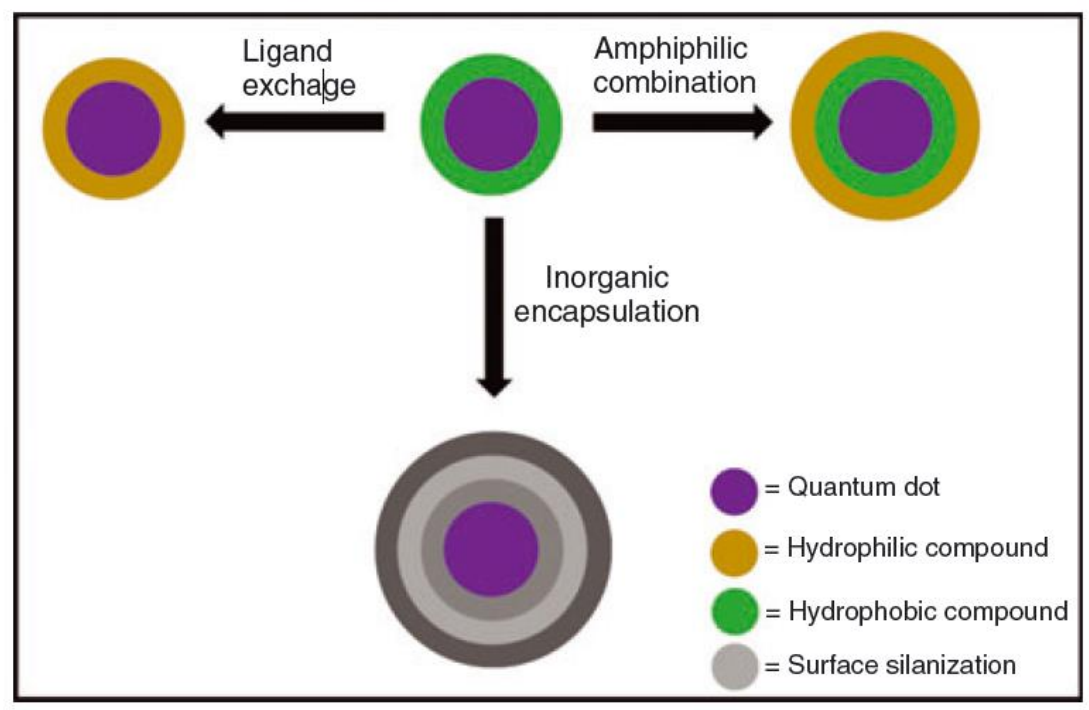

Figure 4. Three commonly used QDs phase-transfer approaches for aqueous stabilization of bare NPs: ligand or cap exchange process, bonding of amphiphilic polymers and phospholipids to hydrophobic groups in the surface of the QDs and surface silanization of the core NP. "Reprinted from Advances in Colloid and Interface Science, 215, Karakoti AJ, Shukla R, Shanker R, Singh S, Surface functionalization of quantum dots for biological applications, 28-45, Copyright (2015), with permission from Elsevier.

As shown in Figure 4, a universal and simple approach is based on the ligand exchange of the QDs original surface hydrophobic coating (e.g. trioctylphosphine oxide chains, TOPO) that is removed and replaced with bifunctional molecules that often binds to the QDs surface (e.g. through a thiol end) having a hydrophilic functional group on the other end (such as carboxyl or sulfonic acids) that provides the required NP solubility in aqueous and polar media and also is available for further bioconjugation [19]. Bidentate ligands as dithiothreitol 
(DTT) or dihydrolipoic acid (DHLA) as well as oligomeric phosphines, peptides, and crosslinked dendrons are widely used to obtain aqueous stable QDs [45].

The second method consists on the well-known silica chemistry used for inorganic encapsulation in hydrophilic structures (e.g. surface silanization) of the QDs through the generation of a silica shell around the NP surface [46]. This is a very attractive approach for water stabilization of QDs as silica surface is non toxic, chemically inert and optically transparent. Typically, in this strategy a precursor molecule such as mercaptopropyltrimethoxysilane (MPTMOS) is added (which replaces the hydrophobic surface chains of the QD). The thiol groups of the MPTMOS react with the inorganic surface of the QDs, and the methoxysilane groups polymerize through formation of siloxane bonds, thus generating a highly cross-linked protective shell around the QDs. Additionally to the previously commented advantages of this approach, silica exhibit a high degree of biocompatibility and its simple to functionalize its surface with appropriate (bio)analyte recognition biomolecules, which make such QDs encapsulated in a silica layer highly suitable for further bioanalytical applications [47].

A third approach to transfer the nonpolar QDs into an aqueous media consist on the use of amphiphilic polymers. Here, the hydrophobic shell of QDs (e.g. TOP/TOPO) interacts with the hydrophobic alkyl chains of the amphiphilic polymeric structures through hydrophobic or electronic interactions. Then, the hydrophilic groups of the amphiphilic polymer used will remain oriented to the external part of the QDs surface, providing the required water stability [48]. There are a wide number of amphiphilic copolymers available that have been used in this approach (e.g. poly(maleic anhydride) [49]) and polyelectrolytes (poly-acrylamide [50], or biopolymers like DNA [51]). Polyethylene glycol (PEG)-based coating of the QDs is another alternative often used to provide stability and biocompatibility to the NPs. Additionally, QDs coated with PEG spacers reduce nonspecific protein binding, which may often limit the applicability of these NPs on the development of bioanalytical methodologies. For such uses, PEG molecules should be previously activated with appropriate functional groups (e.g. amine, thiols or carboxyls) allowing to have available hydrophilic bridges between the QDs surface with the PEG chains [52]. 
A variant of this third stabilization approach consists on the encapsulation of the hydrophobic QDs in appropriate hydrophilic vehicles such as liposomes [53]. The hollow spherical structure of liposomes and the high loading capacity makes them attractive carriers for hydrophobic QDs. Moreover, surface of liposomes can be easily modified with appropriate functional groups to allow a simple further bioconjugation with proteins, minimizing nonspecific interactions of water-soluble and water-insoluble QD with surface material, and amplifying the analytical signal thanks to the possibility to incorporate several QDs in a single nanoliposome. Actually, in this context, quite recently, signal amplification platform based on measurement of fluorescence from QDs encapsulated in liposomes for high sensitive detection of human telomerase activity was proposed [54]. In this approach, similar to a typical hybridization bioassay, biotinylated liposomes containing the QDs were recognized by a capture probe through streptavidin. In a final step, the QD-encapsulated liposomes were disrupted by controlled addition of Triton X-100, and the fluorescence intensity of the released QDs was measured to detect telomerase activity. Liposomes containing hydrophobic QDs have been also employed for tumor imaging applications through the specific recognition of aptamers conjugated to the surface of the liposomes (see figure 5) [55].

It must be considered, however, a significant drawback of liposomes is their low stability when entering in vivo media. Additionally, QDs stabilized by this approach have substantially increased hydrodynamic diameter, which could limit their application in bioimaging and targeting.

It must be taken into account that the procedure selected for hydrophilization of the QDs would most probably affect their subsequent bioconjugation and future bioanalytical application. For example, some approaches can increase significantly the hydrodynamic ratios of the NPs, which can lead to non-specific binding or reduced accessibility to some targets. In this context ligand exchange provides QDs with a small hydrodynamic diameter but also with lower photoluminescence quantum yields, while encapsulation results in larger nanoprobes sizes with higher quantum yields [56]. 
(a)
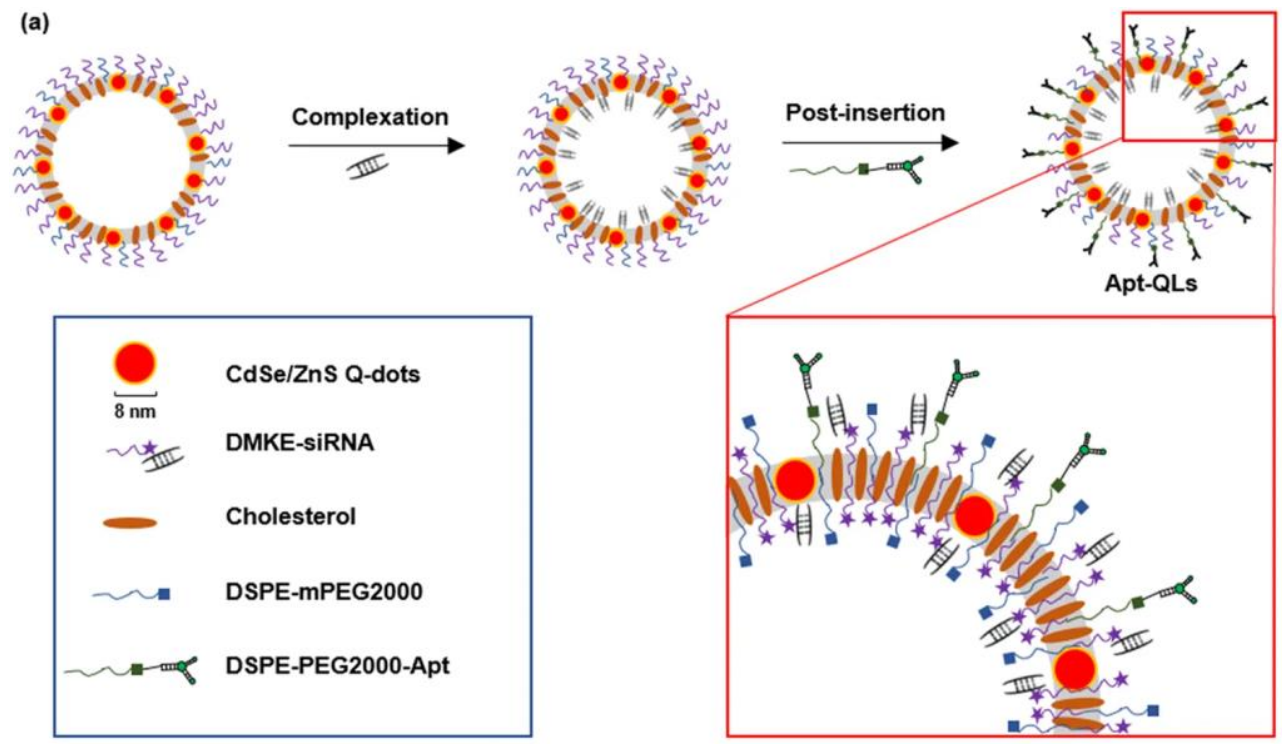

(b)

(c)

(d)
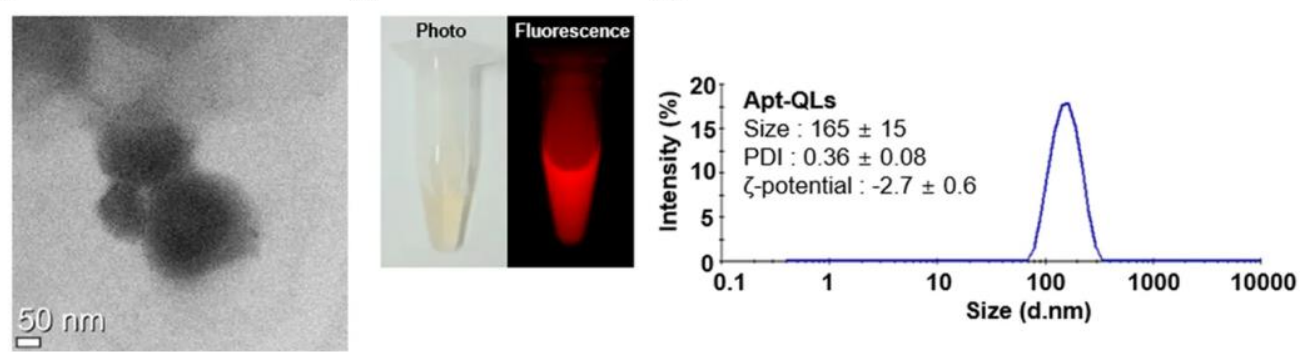

Figure 5. (a) Schematic illustration of the synthesis of liposomes containing QDs and an aptamer against the EGF receptor. (b) The liposomes containing QDs were visualized by TEM, (c) fluorescence emission from was verified $\left(\lambda_{\text {ex }} 550 \mathrm{~nm}, \lambda_{\mathrm{em}} 620 \mathrm{~nm}\right),(\mathbf{d})$ and size obtained using Dynamic Light Scattering gave rise to $165 \mathrm{~nm}$ diameter liposomes. Reprinted from Scientific Reports, 7, Article number: 9474, Kim MW, Jeong HY, Kang SJ, Choi MJ, You YM, Im CS, Lee TS, Song IH, Lee CG, Rhee KJ, Lee YK, Park YS, Cancer-targeted Nucleic Acid Delivery and Quantum Dot Imaging Using EGF Receptor Aptamer-conjugated Lipid NPs, Copyright (2017).

\section{QUANTUM DOT BIOCONJUGATION STRATEGIES}

Probably, the main challenge for the use of QDs in biomedical applications is the robust bonding of appropriate target recognition molecule on the NP surface, which will be a key parameter affecting their direct application in biological media. In this section, we will 
summarize some of the most relevant physicochemical processes used to attach the biomolecules (BMs) to the QD surface, a process named bioconjugation.

All the NPs have a very high surface-to-volume ratio. Under these circumstances, the role of the NP surface becomes of paramount importance [57]. In fact, its properties are determined not only by their own chemical nature, but also by the layer of capping molecules, named ligands because they bind to the QD surface metals, in a similar way as the ligands do to the central atom in the metal complexes. Thus, ligands "stop particles aggregating, resist nonspecific adsorption of surrounding molecules, and provide a conjugation point for functional biomolecules" [58]. The synthetic method utilized for the preparation of QDs determines not only its size, shape and chemical nature, but also the ligands capping its surface. In fact, these ligands are chosen mainly because they have to control the size, shape and polydispersity of QDs during the synthesis, and to maintain their homogeneous dispersion in the solvent after the preparation. Ligand exchange reactions extend the versatility of QD material.

The main items to which it should be paid attention during the bioconjugation process have been described in a comprehensive article [56] and can be (1) Control over the biomolecule/ $Q D$ ratio, being the desirable ratio variable with the type of the application. It should be noted that QDs are usually bigger than BMs, except, perhaps some large proteins. Furthermore, it should be taken into account that the reactions are interfacial in their nature and such interfaces are inherently polydisperse across a population of QDs. (2) Control over orientation of the $B M$ on the $Q D$, maintaining the optimal activity of both the QD and the BM. (3) Control over the separation between $Q D$ and $B M$. This point is crucial if the platform QD-BM is to be used in a FRET (Förster resonance energy transfer) experiment [59]. (4) Control over the strength of the QD-BM bond. Most of the clinical or in vivo experiments require permanent, and therefore, strong linkage.

Different approaches can be used to immobilize biomolecules onto the QD surface. However, the simplest way to link a biomolecule to a QD surface is by adsorption. In the adsorption, the attachment of BM is rather tenuous and it is maintained by weak interactions like hydrogen bonding, the London dispersion and the Coulombic forces, and lone-pair electrons [60]. In fact, the proteins present in the human body tend to bind nonspecifically 
onto the QDs surface, which is something to be avoided. Closed related are the pure electrostatic interactions between the BM and the QD [61]. Although this method of functionalization is generally straightforward and fast, the electrostatic interaction, like the simple adsorption, suffers from serious disadvantages: instability, lack of orientation control on the biomolecule and on the ratio BM/QD, etc. [62,63]. Actually, the bioconjugation usually follows one of the four different routes depicted in Figure 6 [56].

\subsection{Direct union of $B M$}

The first one is the direct covalent union of biomolecules to the surface of the QD semiconductors. Thus, proteins, peptides and nucleic acids could be bonded to the QDs surface metal atoms (especially $\mathrm{Zn}$ ) through their (cysteine) thiol and the (hystidine) imidazole groups [56]. The thiol and hystidine motifs could be eventually added to the "natural" biomolecules, and, occasionally the thiol groups are created reducing peripheral S$\mathrm{S}$ bonds. Occasionally, the biomolecules are bonded directly to the QD in the synthesis processes (biological templating) [56].

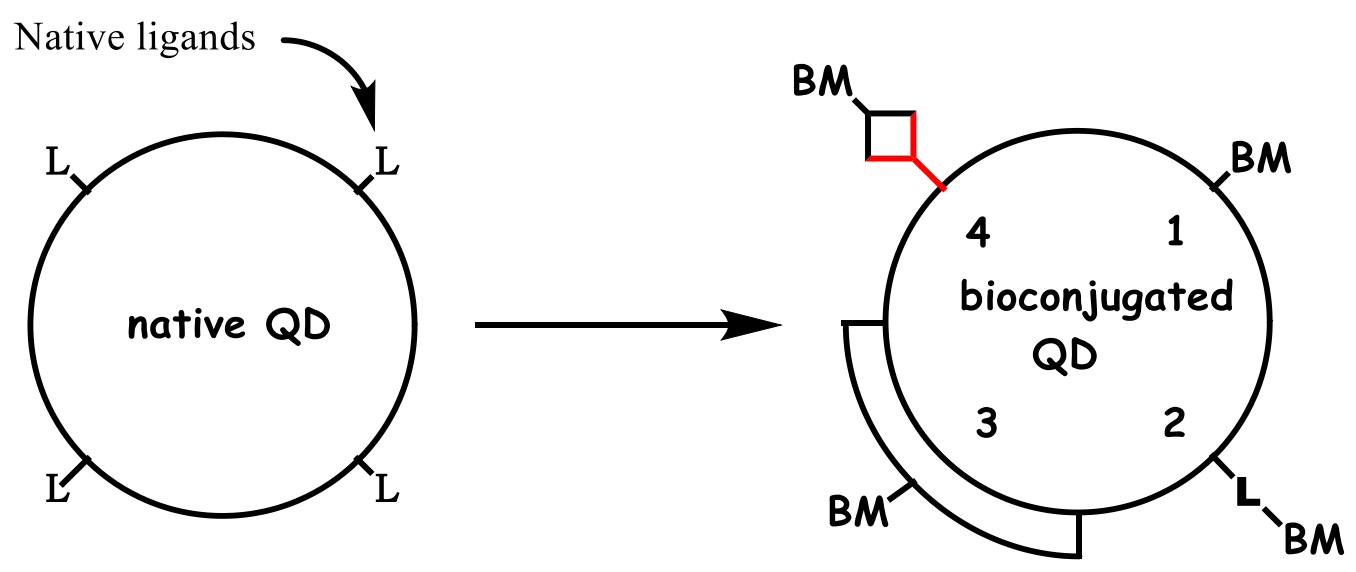

\section{The four main routes to} bioconjugugation

\section{1: direct union}

2: $B M$ bonded to a ligand $L$

3: $B M$ bonded to an encapsulating shell 4: BM bonded through Biotin/Sreptavidin

Figure 6. The four main routes to join a biomolecule, BM, to a semiconductor QD surface. 1) By direct covalent bond. 2) Through a covalent bond between the BM and a ligand L, previously 
anchored on the QD surface. 3) Binding covalently the BM to a terminal functional group integrated in an encapsulating shell. 4) Taking advantage of the specific union Biotin/Streptavidin.

\subsection{BM bonded to a ligand}

The second possibility, more general, is to bind covalently the biomolecule to a ligand previously attached to the QD surface (See Figure 6). In this regard, it should be pointed out that Graphene QDs (GQDs) usually contain carboxyl, hydroxyl, carbonyl and epoxide external groups [64] and Carbon QDs (CQDs) can be synthesized to have selectively carboxylic, amine or other groups in their surface [65]. On the contrary, native semiconductor QDs, with some exceptions prepared in water [66,67] do not have appropriate ligands able to be robustly bonded to BM. However, this is not a problem because, as seen in the previous section, the original ligands can be replaced by others. The new ligands could be monodentate or bidentate simple molecules like 3-mercaptopropionic acid (MPA) or dihydrolipoic acid (DHLA) respectively [68] attachable to the QDs surface through the thiol group(s) and possessing a free terminal carboxylic group to join the BM. However, the new ligands are usually complex molecules with an anchoring group(s) (e.g. polythiol), a spacer chain (frequently a hydrophilic segment) and a terminal functional group (carboxylic or amine groups inter alia) [69]. Very often, the spacer chain is the polyethylene glycol, PEG, that is, the whole ligand is a bifunctional PEG molecule [67] because PEG is biocompatible, high soluble in water and stabilize QD against aggregation.

\subsection{BM bonded to an encapsulating shell}

The native ligand exchange has some problems, the weaker bond between the thiol group and the metal of the QD surface, the reduced photoluminiscent quantum yield, etc. [61, 63]. An alternative is the encapsulation of the QDs, (the third route shown in Figure 6) either with a layer of amorphous silica, or with a copolymer.

The formation of a silica outer sphere (silanization) increases the solubility and stability of the QDs and retains most of its emission properties [63]. It has been described different silanization processes $[63,67,70,71]$. In general, they are laborious and require several steps 
[63]. The silica layer is bonded to the QD surface through an anchoring group, mostly thiol or amino groups (A in Figure 7A) and at the periphery it possesses also functional groups, thiol and amino inter alia, ( $\mathrm{F}$ in Figure 7A).

The encapsulation of a semiconductor QD with amphiphilic copolymer is possible thanks to the ability of its long hydrophobic tails to interact and interdigitate with the pristine QD ligands (like trioctylphosphine, trioctylphosphine oxide, hexadecylamine, stearic acid, etc.)[67] leaving the hydrophilic and functionalized segments in contact with the water molecules of the solvent $[61,63,70]$. Although the copolymers based in maleic anhydride or in acrylic acid are the most popular, $[61,63,70,72,73]$ there are some other examples, for instance, the phospholipid-PEG copolymers, the last possessing an terminal functional group [74] (See Figure 7B).

A) SILICA SHELL

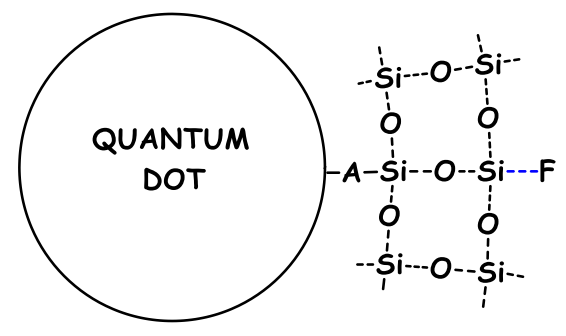

B) COPOLYMER ENCAPSULATION

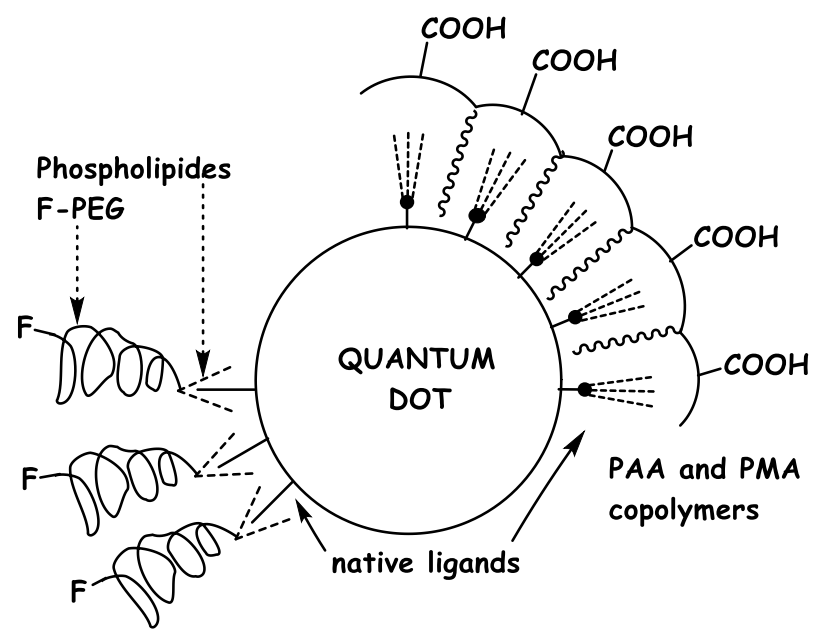

Figure 7. Encapsulation of semiconductor QD: (A) with silica shell or (B) with copolymers. The silica layer binds to the QD surface through an anchoring group A and possesses functional groups $\mathrm{F}$ in the periphery. The copolymers more frequently used for encapsulation are Poly(maleic anhydride) (PMA) or Poly(acrylic acid) (PAA) based copolymers but also the end-functionalized polyethylenglycol F-PEG-Phospholipides. 


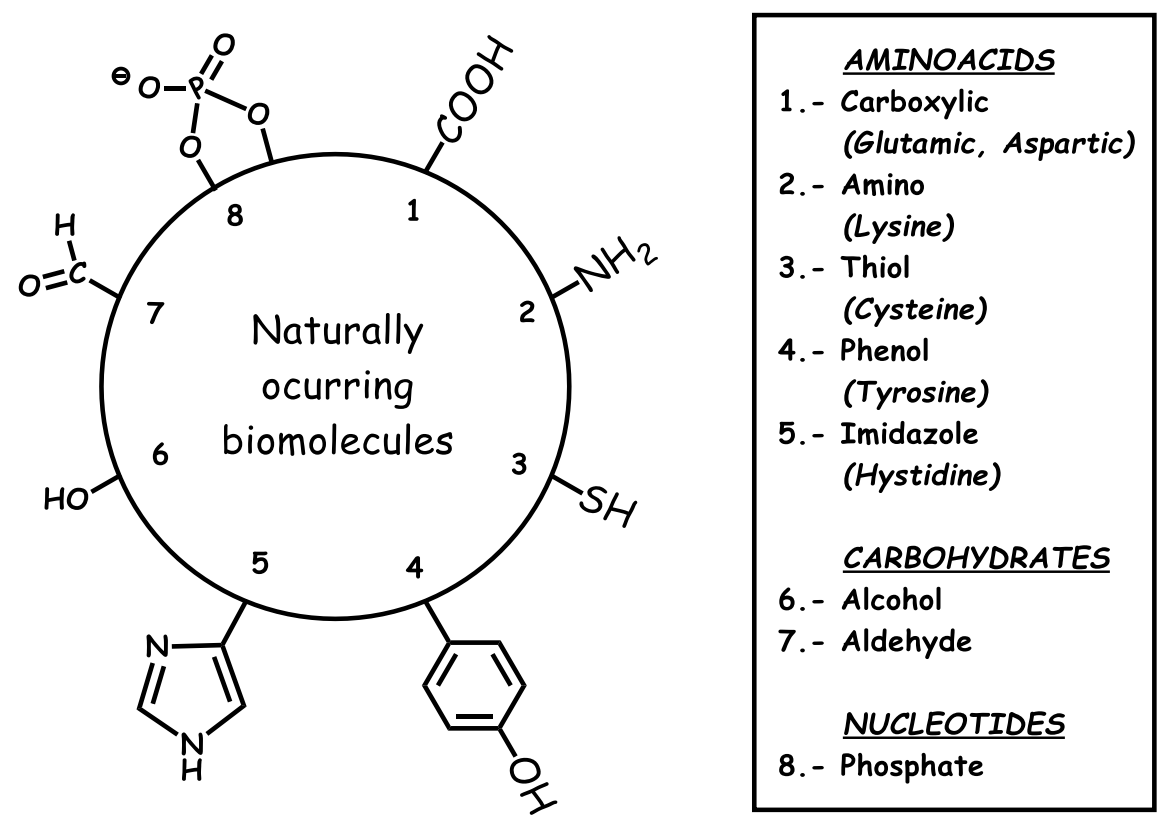

Figure 8. The most important functional groups found in the natural occurring biomolecules: carboxylic, amino, thiol, phenol, imidazole (present in aminoacids); alcohol and aldehyde (coming from carbohydrates) and phosphate (component of nucleotides)

Some authors consider the envelopment of several QDs inside a polyethylenimine coat [75] or inside the bilayer of a liposome [76,77], as particular cases of QD encapsulation. Although these examples are not strictly comparable to those described in Figure 7, in which every QD is singularly encapsulated, certainly there exists some similarity.

\subsection{BM bonded trough Biotin/Streptavidin}

Other method used very frequently to join biomolecules to the QD surface is based in the biotin-avidin strong interaction, with a dissociation constant of $10^{-15} \mathrm{M}$ [78]. Avidin, a protein found in the egg white, contains four identical subunits, each with a single biotin-binding site. Biotin is the vitamin H. Usually, Avidin is advantageously substituted by deglycosylated Avidin derivatives like Streptavidin or Neutravidin [56,62]. There are a variety of biotin derivatives that make biotinylation of QDs and biomolecules rather straightforward $[56,62]$. QDs and BMs may be also functionalized with avidin, although it should be noted that the attachment of avidin to the QD during initial modification will probably obscure one or more 
available biotin binding sites [56]. Later, avidin-functionalised QDs could be attached to biotinylated biomolecules. Conversely, Biotin-functionalised QDs can be used to join Avidin-functionalized biomolecules. Even Biotin-labeled QDs can couple to Biotin-labeled biomolecule by an intermediary avidin linker [56,61]. However, in the last case it is possible to obtain unexpected results, since the avidin can bind up to four biotin moieties $[56,61]$.

There exists more types of specific non-covalent affinity between pairs of molecules other than Avidin/Biotin interactions that are useful to bioconjugation: Histidinenickelnitrilotriacetic acid interaction, Barnase-barstar interaction, and Antibody-ligand interaction [62].

\subsection{Covalent coupling strategies}

We shall center now in the covalent bonding between the ligands anchored on the QDs surface (directly or attached to an encapsulating shell) and the incoming biomolecules. In this regard, we consider Biotin and Avidin (or Straptividin or Neutravidin) as BMs.

The number of functional groups useful for bioconjugation reactions is rather limited in natural BMs [56]. (See Figure 8). Carboxylic and amino groups are present in peptides and proteins not only as terminal groups but also as side groups in peptides like aspartic or glutamic acids or lysine respectively. Less habitual in peptides and proteins are the thiol, phenol and imidazole groups that can be found in the aminoacids cysteine, tyrosine and hystidine, respectively. Other functions that are available in even more infrequent aminoacids like tryptophan will not be taken into account. Carbohydrates and their derivatives provide alcohol and aldehyde as reactive groups, the latter are obtained by oxidation of the former. Nucleic acids possess sugars, phosphates and some bases (the bases were not included in Figure 8 because they are not usually modified).

Although the available BMs functional groups would appear scarce, the amount of possible reactions to join covalently the BMs to the ligands attached to the QDs described in the literature and even in the commercial sources is rather numerous [56,62,63,71,76,79-81]. However, only a small portion of them seem to have been used actually when QDs are involved $[62,63,82]$. 
One of the most well-studied and easy-to-perform is the reaction, in mild conditions, between a terminal carboxylic acid and a peripheral amine group to yield an amide group, with the help of EDC (1-ethyl-3-(3-dimethylaminopropyl) carbodiimide hydrochloride) and sulfo-NHS (N-Hydroxysulfosuccinimide sodium salt). In spite of all the disadvantages that this method presents, it is still widely used $[62,63,82]$. An alternative is to use Carbonyldiimidazol (CDI) instead of the EDC/NHS couple [63,83].

Other common routes are the reaction of amines with carbonyl groups to yield an imine groups, which usually are subsequently reduced with sodium cyanoborohydride $[62,63]$, and the Michael addition of a terminal thiol to a maleimido group [63,80]. Another popular coupling method is the utilization of heterobifunctional molecules like sulfosuccinimidyl-4(N-maleimidomethyl) cyclohexane-1-carboxylate (sulfo-SMCC). The NHS ester end of sulfo-SMCC can react with primary amine groups and the other terminal maleimido function can add a thiol $[62,63]$. Similar crosslinker molecules are described in the literature $[56,62,80]$.

\subsection{Bioorhogonality}

The traditional coupling methods so far mentioned have some limitations; mainly, undesirable side reactions [56]. The solution to this and other problems is the so called bioorthogonal chemistry. The bioorthogonal reactions involve only the target functions (in QD and BM) and do affect the other functional groups present neither in the affected QD and $\mathrm{BM}$ nor in the biological environment $[84,85]$.

Again, although there are numerous biorthogonal reactions described in the literature [8486], only some of them seem to have been carried out when the QDs are involved, namely, those described in Figure 9. That is, the copper-catalyzed alkyne-azide cycloaddition (click chemistry); the cycloaddition between tetrazine and strained double bonds (tetrazine ligation), and the hydrazone formation by reacting hydrazine and carbonyl groups (hydrazine ligation). It should be pointed out that no one of the functional groups shown in Figure 8, those which are present in the "natural" biomolecules, is implicated in these bioorthogonal reactions. This is, of course, the most important advantage of the bioorthogonal chemistry; the reactions described in Figure 9 could not affect normal molecules present in the biological 
milieu. Certainly, it would be necessary to look for some procedures to attach these "new" functional groups to the natural BM since it is not foreseeable find any trouble to bind them to the QDs. In this regard,a it should be said that it has been found that virtually any functional group can be site-specifically introduced in peptides and nucleotides as needed during initial synthesis or by subsequent modification [56].

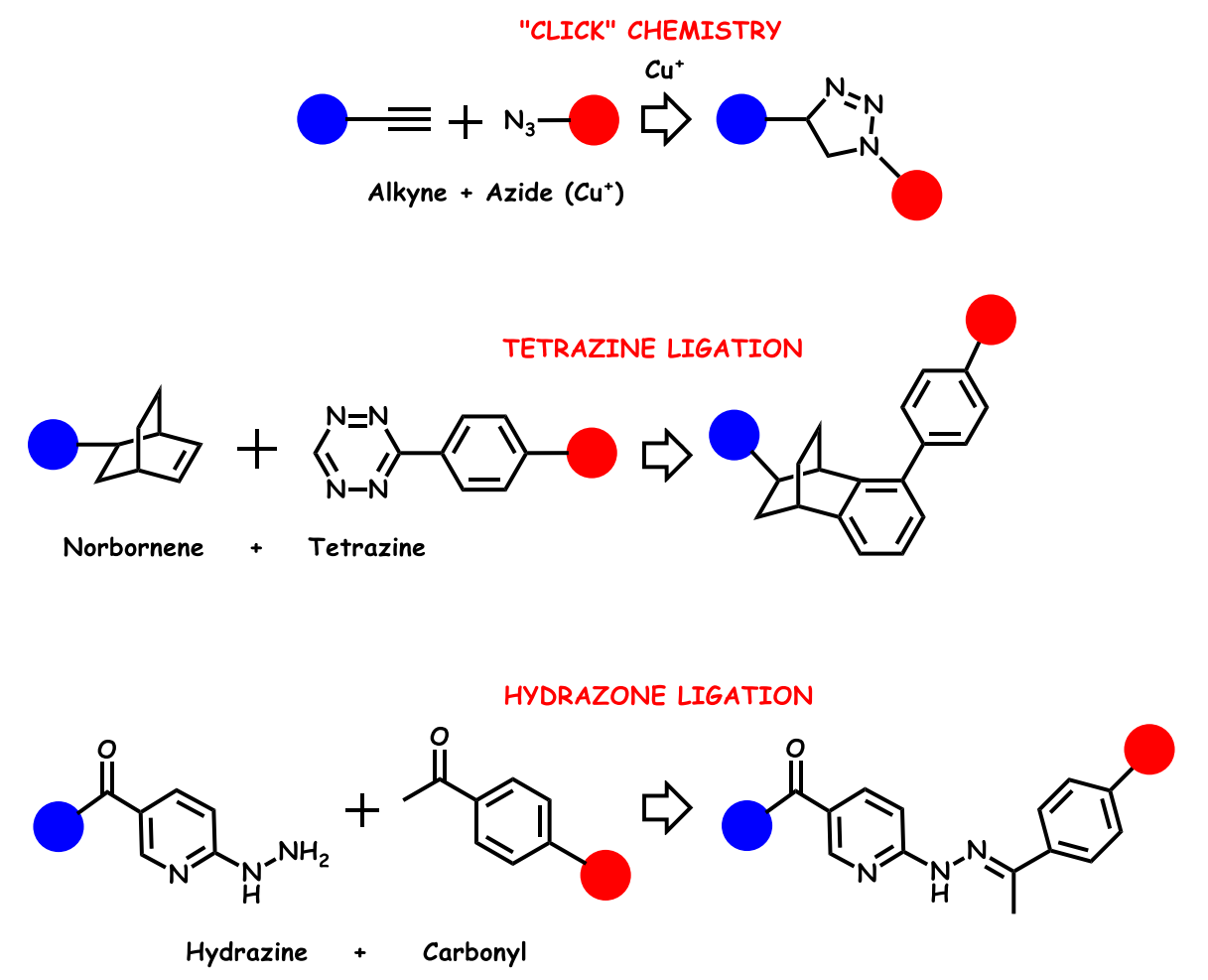

Figure 9. The main bio-orthogonal reactions carried out utilizing QDs: copper-catalyzed alkyneazide cycloaddition; cycloaddition between tetrazine and strained double bonds, and hydrazone formation by reacting hydrazine and carbonyl groups

\section{QDS BIOSENSING APPLICATIONS: POINT OF CARE (POC) DIAGNOSTICS}

QD-biomolecule hybrids (bound to antibodies, DNA, aptamers, etc.) have been used as sensing probes in different types of in vitro bioassays and biosensors for the detection of different clinical relevant biomolecules. QDs as labels can be used with a variety of detection methods (Figure 10). Most of the reported QD-based bioassays and biosensors have been 
developed by using QDs as fluorescence labels (Figure 10a) in fluorescence quenching-based (turn-off), fluorescence enhancement-based (turn-on), and especially in fluorescence resonance energy transfer-based (FRET) assays [87-92]. FRET is a very sensitive technology to study biomolecule interactions that involves the transfer of energy from an excited state donor (usually a fluorophore) to a proximal $(<10 \mathrm{~nm}$ ), ground state acceptor (Figure 10b).

Semiconductor QDs (SQDs) have been by far the most reported type of QDs applied in this field [93]. Main advantages of SQDs over the organic dyes typically used as fluorescence labels include long fluorescence lifetime, broad absorption spectra, very narrow emission spectra and stability against photobleaching [94]. Another interesting advantage of SQDs is their size-controlled luminescence (Figure 10a). This property allows for the simultaneous determination of different biomolecules by using a single excitation wavelength $[95,96]$. However, the multiplexing capability of SQD as optical labels is quite limited (5-6 biomolecules). Greater multiplexing capabilities have been obtained by embedding differentsized quantum dots with different intensity levels into polymeric particles at precisely controlled ratios [97,98]. This strategy provides a quantum dot barcode technology able to create more than million codes in theory (Figure 11). Main limitations of SQDs include their low long-term stability, intrinsic blinking, complex synthesis and complexation and high cost of production.

As stated above, in addition to fluorescence assays, electrochemiluminescence (anodic/catodic) (Figure 10c), photoelectrochemical (anodic/catodic) (Figure 10d) and especially electrochemical (Figure 10e) QD-based biosensing systems have also been reported [99-101]. Electrochemical devices have emerged as the main alternative to fluorescence assays thanks to their high sensitivity and their simple and inexpensive instrumentation [100,101]. As their optical counterparts do, most of the reported electrochemical QD-based bioassays and biosensors rely on the use of SQDs as electroactive labels. SQDs have been exploited for electrochemical detection on the basis of their elemental compositions. Stripping voltammetry has been the electroanalytical technique most widely employed to quantify the metal ions released upon SQDs solubilization [102105]. Based on the different chemical composition, SQDs have been used as electroactive labels for multiplexed analysis [106]. 

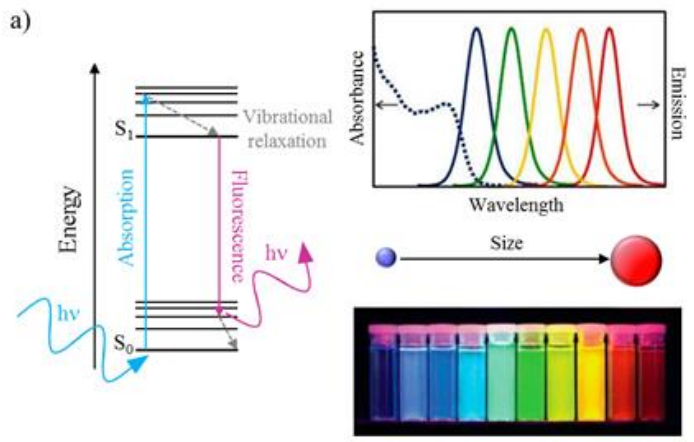

b)

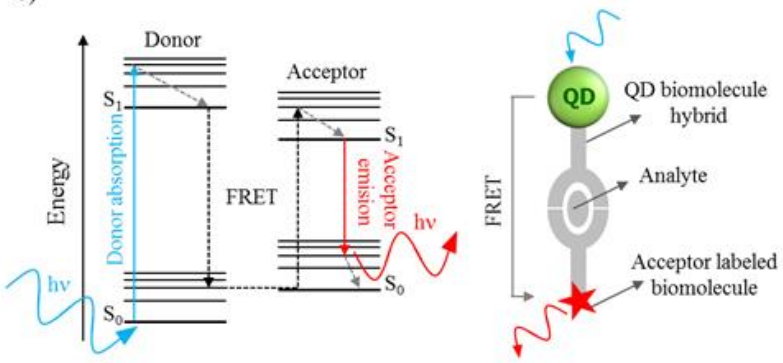

c)

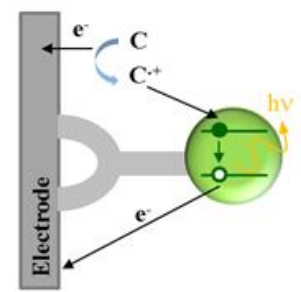

d)

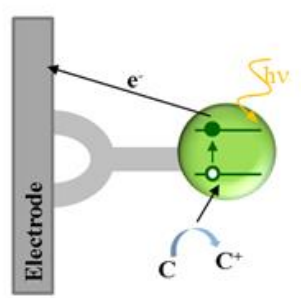

e)

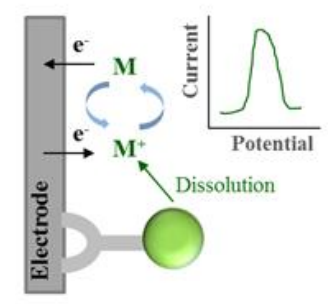

Figure 10. Detection methods for QD-based biosensing: a) Fluorescence: Jablonski diagram explaining the effect of fluorescence (left), and photograph and emission spectra illustrating size controlled fluorescence of QDs (right, [29]. Reprinted with permission from reference 21. Copyright 2010 Wiley. b) FRET: Jablonski diagram explaining the effect of FRET (left) and schematic illustration of a FRET based sandwich bioassay (right); c) Electrochemiluminescence (ECL): schematic illustration explaining anodic ECL. A hole is created by the electrode in the valence band of QDs with the concomitant injection of an electron from a previously oxidized coreactant $(\mathrm{C})$. The recombination of electron and hole lead to an anodic ECL emission; d) Photoelectrochemical (PEC): schematic illustration explaining anodic PEC. An electron-hole pair is created on QDs after their photoexcitation. The electron transferred from the valence band to conduction band of photoexcited QDs is then ejected to the electrode with the concomitant transfer of electrons from an electron donor (C), generating an anodic photocurrent; e) Electrochemical (EC), schematic illustration explaining 
the electrochemical detection of QDs by anodic stripping voltammetry. After the dissolution of QDs, metallic species are deposited (reduced) on the electrode and re-oxidized again to be detected.

a)
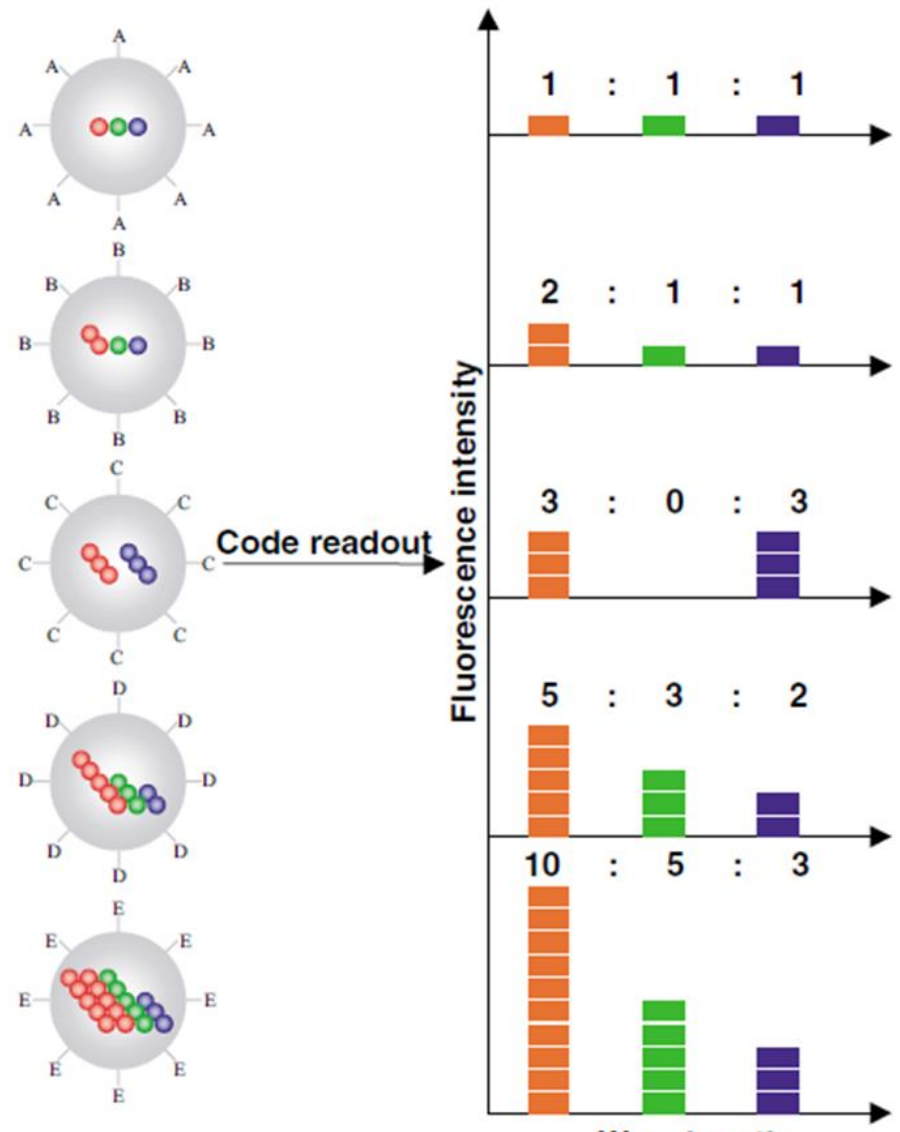

Wavelength

b)

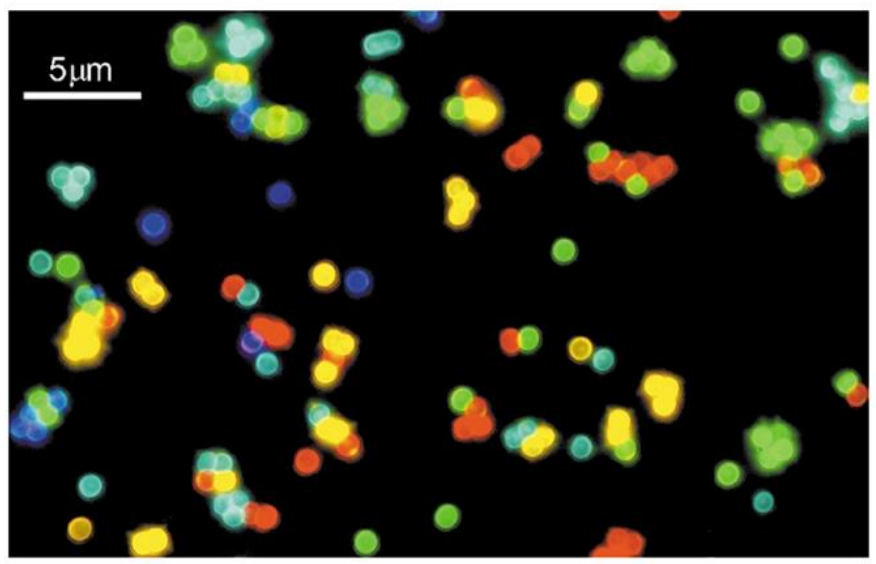


Figure 11. QD barcode technology. a) Schematic illustration showing optical encoding scheme using wavelength and intensity via multiple QDs embedded within microparticles; b) Color micrograph showing microbeads. Reprinted with permission from [97]. Copyright 2001 Nature.

Regardless of the detection method used for QDs based biosensing, most of the reported systems are time-consuming, have to be performed in centralized laboratories by highly skilled personnel, and are therefore not suitable for "point-of-care" (POC) diagnostics. Modern healthcare systems are demanding diagnostic platforms for real-time remote monitoring of health biomarkers to adopt a more patient centered approach to care [107]. So, the development of simple and cost-effective POC diagnostic systems able to obtain useful information instantly at the sampling site has been the focus of biosensing research over recent years.

Microfluidic systems have become an increasingly attractive alternative to the centralized laboratory assays for POC applications. Microfluidic devices allow conventional assays to be performed using an automated and high-throughput approach offering advantages such as small reagent consumption, low-cost, portability and short analysis time. Examples of onchip single and multiplexed assays for medical diagnostics based on SQDs with optical and electrochemical detection can be found in literature [108-113]. Nevertheless, the performance of most of these microfluidic devices required the implementation of bulky and energy consuming off-chip fluidic handling components (pumps, valves) and nonminiaturized detectors, making them not suitable for POC measurements. In this context, paper-based devices have emerged as a class of microfluidic devices that can work without the need of any off-chip fluidic handling element, showing therefore a significant potential for POC applications [114]. In these devices the liquids are driven by capillary forces without requiring external components. Furthermore, compared to silicon, glass and other polymeric materials used for the fabrication of microfluidic chips, paper is cheap, biodegradable, widely available, flexible and easy to be modified with biomolecules. In spite of limitations in terms of sensitivity and reproducibility, paper-based devices are claimed to be the future of pointof-use testing, thanks to their simplicity, low-cost and disposability. 
In recent years, smartphone devices, which have millions of users worldwide including third-world regions, have been adapted to tackle limitations in the instrumentation used for POC applications. Owing to their low-cost, functionalities, accessibility, small size and easeof-use, the integration of smartphones in the development of easy-operable POC devices is very promising [115]. Moreover, the connectivity of smartphones gives the possibility to share data through the cloud for backup or remote processing of the results, which is highly demanded for modern healthcare systems.

Recent advances on QD-based microfluidic paper devices and QD barcode POC applications are discussed in detail in the flowing sections.

\subsection{QD-based microfluidic paper devices}

Paper-based devices can be classified as dipsticks assays, lateral flow assays (LFAs) and microfluidic paper analytical devices ( $\mu$ PADs). The dipstick assays are the simplest paperbased devices. They are based on a paper strip pre-stored with reagents that is dipped into a sample to perform a chemical test (e.g. pH strips). Lateral flow assays (LFAs) are the most widely reported form of paper devices (e.g. pregnancy test) [116]. The basic structure of a lateral flow strip is composed of four different parts that are fixed in a backing card (Figure 12a): a sample pad, on which the sample is dropped; a conjugation pad, on which labelled tags conjugated to the biorecognition elements are immobilized; a reaction membrane which contains test and control lines for reactions; and an absorbent pad, which reserves waste and prevents backflow. Briefly, when a sample is added on the sample pad, the sample flows via capillary forces towards the end of the strip. If the analyte is present, the immobilized bioconjugate on the conjugation pad binds to the analyte and continue migrating along the test. As the sample moves along the device, the binding reagents placed on the reaction membrane bind to the analyte at the test line. Bioconjugates free of analyte finally react with specific bioreceptors immobilized on the control line of the membrane (Figure 12b). $\mu$ PADs

are the more complex but more versatile paper devices [117]. In these devices the formation of hydrophilic channels with hydrophobic barriers enabling multidirectional and multidimensional flow which allows for complex bioassays to be performed. 
Most of the reported paper devices rely on the use of gold NPs (AuNPs) as labels, with qualitative (naked-eye) or semi-quantitative colorimetric detection. Main limitations of colorimetric detection of AuNPs include limited quantitative dynamic ranges and low sensitivity, even with reader systems [118,119]. In this context, the use of QDs as fluorescence labels is becoming increasingly popular. Fluorescence detection is a good choice to improve both the limit of detection and the dynamic range of colorimetric paperbased devices. However, an important drawback of fluorescence detection for POC applications is the need of complex reader systems for the results interpretation. 
a) Labeled biomolecule Primary biomolecule Secondary

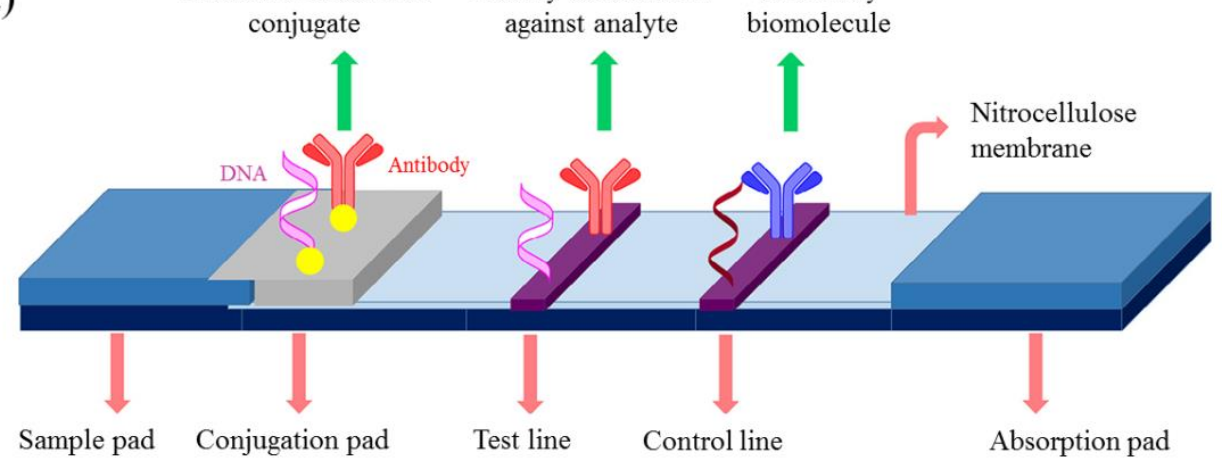

b)

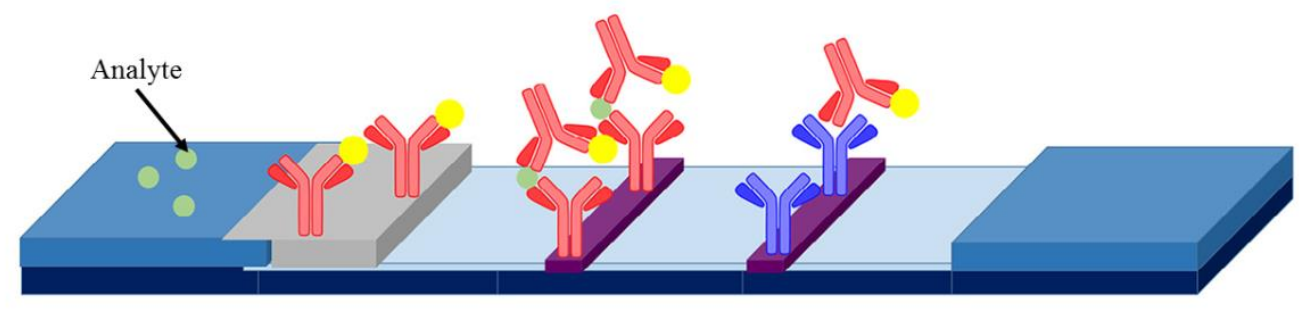

c)
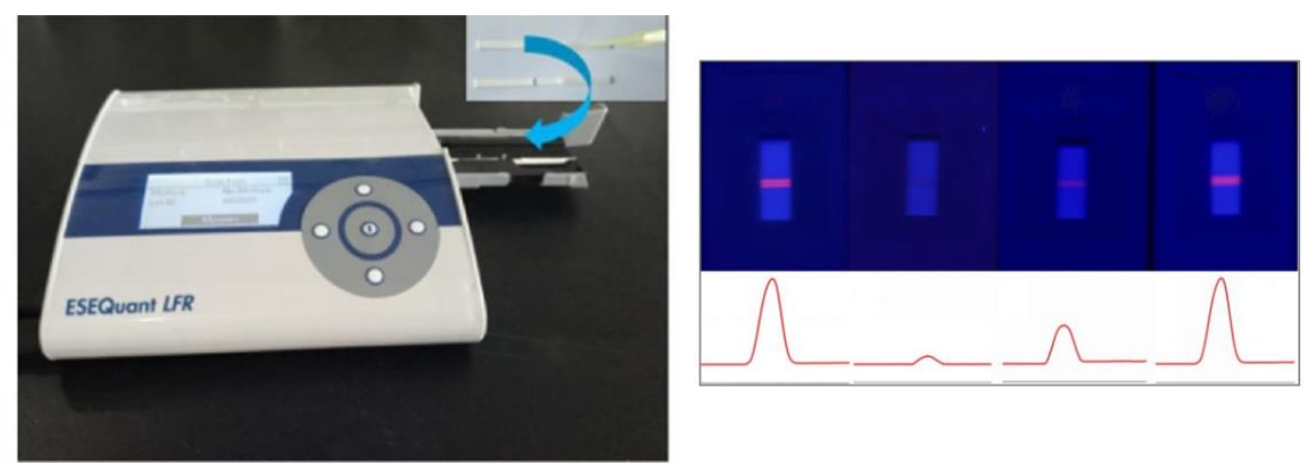

Figure 12. QD -based LFAs. Schematic representation of: a) The basic structure of a LFA; b) Sandwhich immunoassay format LFA (Reprinted with permission from [116]. Copyright 2016 Elsevier); c) Photograph of a portable fluorescence test strip reader and images of the QDs-based fluorescence test strips with different concentrations of the analyte (gluthatione) together with the curves obtained from the reader (Reprinted with permission from [121]. Copyright 2019 Elsevier). 
SQDs have been used as signal reporters in the development of different types of paper based biosensors. Sapountzi et al. [120] designed the first SQD-based dipstick for visual detection of nucleic acids and single nucleotide polymorphisms (SNPs) in human genomic using a common digital camera and a UV lamp for fluorescence imaging. As low as 1.5 fmol levels of double-stranded DNA were clearly detected by naked eye using CdSe/ZnS coreshell QDs as signal reporters. The dipstick performance was accurate and reproducible, being also successfully applied to real sample analysis.

Examples of single and multiplexed fluorescence-based LFAs for a wide variety of clinical relevant biomolecules using QD-hybrids (conjugated to protein or DNA) as signal reporters can also be found in literature (Table 1) [121-129]. As shown in Table1, the use of QDs as labels in the development of LFAs allowed for fast and sensitive determination of different protein biomarkers and DNA. Such QD-based LFAs exhibited similar performance than conventional methods [121-124] and were successfully applied for biomolecules determination in real samples.

QD micro/nanospheres have also been employed as fluorescent labels for LFAs. These spheres, prepared by embedding a large amount of QDs into polymeric or silica beads, yield a substantially enhanced fluorescence signal, resulting in an increased sensitivity. Following this strategy, Rong et al. [130] reported a smartphone-based fluorescent LFA for highly sensitive and selective detection of Zika virus nonstructural protein 1, using CdSe/ZnS QDs encapsulated in polymer microspheres as labels (Figure 13a). Within only $20 \mathrm{~min}$, the optimized sandwich lateral-flow immunoassay achieved sensitive detection of Zika protein with LODs of 0.045 and $0.15 \mathrm{ng} \mathrm{mL}^{-1}$, in buffer and serum respectively.

QD nanobeads were used by Li et al. [131] in the development of a LFA for prostate specific antigen (PSA). QD nanobeads (60 $\mathrm{nm}$ in diameter) were fabricated by encapsulating $\mathrm{CdSe} / \mathrm{CdS} / \mathrm{Cd}_{\mathrm{x}} \mathrm{Zn}_{1-\mathrm{x}} \mathrm{S} / \mathrm{ZnS}$ QDs with modified poly(tert-butyl acrylate-co-ethyl acrylate-comethacrylic acid). A very sensitive (LOD $0.33 \mathrm{ng} / \mathrm{mL}$ ) and selective response to PSA was obtained in only 15 min using a portable fluorescence test strip reader. This sandwich lateralflow immunoassay was also successfully evaluated in clinical serum samples.

Table 1. Fluorescence QD-based LFAs. 


\begin{tabular}{|c|c|c|c|c|c|}
\hline Analyte(s) & QD & Assay format & Assay time/ LOD & Real sample & Ref. \\
\hline $\begin{array}{l}\text { Heart fatty acid binding } \\
\text { protein }\end{array}$ & $\mathrm{CdTe}$ & $\begin{array}{l}\text { Sandwich } \\
\text { immunoassay }\end{array}$ & $15 \mathrm{~min} / 221 \mathrm{pg} \mathrm{mL}^{-1}$ & Human serum & [114] \\
\hline $\begin{array}{l}\text { C-reactive protein (CRP) } \\
\text { Interleukin-6 (IL-6) }\end{array}$ & CdTe & $\begin{array}{l}\text { Sandwich } \\
\text { immunoassay }\end{array}$ & $\begin{array}{l}30 \mathrm{~min} / 0.3 \mu \mathrm{g} \mathrm{mL}^{-1} \\
(\mathrm{CRP}) \\
\quad 0.9 \mathrm{pg} \mathrm{mL}^{-1} \text { (IL-6) }\end{array}$ & Human serum & [115] \\
\hline $\begin{array}{l}\text { Carcinoembryonic } \\
\text { antigen (CEA) }\end{array}$ & $\mathrm{CdSe} / \mathrm{ZnS}$ & $\begin{array}{l}\text { Sandwich } \\
\text { immunoassay }\end{array}$ & $15 \mathrm{~min} / 0.049 \mathrm{ng} \mathrm{mL}^{-1}$ & Human serum & [116] \\
\hline Glutathione & CdSe@ZnS & $\begin{array}{l}\text { Displacement } \\
\text { assay }\end{array}$ & $10 \mathrm{~min} / 25 \mathrm{nM}$ & $\begin{array}{l}\text { Hela cells } \\
\text { extract samples }\end{array}$ & [117] \\
\hline $\begin{array}{l}\text { Gastric cancer } \\
\text { carbohydrate antigen } 72-4\end{array}$ & $\mathrm{CdSe} / \mathrm{ZnS}$ & $\begin{array}{l}\text { Sandwich } \\
\text { immunoassay }\end{array}$ & $10 \mathrm{~min} / 2 \mathrm{IU}^{2} \mathrm{~mL}^{-1}$ & Human serum & [118] \\
\hline $\begin{array}{l}\text { Human } \\
\text { Immunodeficiency Virus- } \\
\text { DNA }\end{array}$ & $\mathrm{CdTe}$ & $\mathrm{SDA}^{1}$ & $15 \mathrm{~min} / 0.76 \mathrm{pM}$ & Human serum & [119] \\
\hline $\begin{array}{l}\text { Influenza A } \\
\text { Nucleoprotein }\end{array}$ & $605 \mathrm{~nm}$ QDs & $\begin{array}{l}\text { Sandwich } \\
\text { immunoassay }\end{array}$ & $1.5 \mathrm{fmol}$ & - & [120] \\
\hline \multicolumn{6}{|l|}{ Simultaneous } \\
\hline Alpha fetoprotein (AFP) & $546 \mathrm{~nm}$ QDs & \multirow{2}{*}{$\begin{array}{l}\text { Sandwich } \\
\text { immunoassay }\end{array}$} & $15 \mathrm{~min} / 3 \mathrm{ng} \mathrm{mL}^{-1}(\mathrm{AFP})$ & \multirow{2}{*}{ Human serum } & \multirow{2}{*}[121]{} \\
\hline $\begin{array}{l}\text { Carcinoembryonic } \\
\text { antigen (CEA) }\end{array}$ & $620 \mathrm{~nm}$ QDs & & $2 \mathrm{ng} \mathrm{mL} L^{-1}(\mathrm{CEA})$ & & \\
\hline Simultaneous & \multirow{2}{*}{$\begin{array}{l}\mathrm{CdSe} / \mathrm{ZnS} \\
\text { QDs }\end{array}$} & \multirow{2}{*}{$\begin{array}{l}\text { Sandwich } \\
\text { immunoassay }\end{array}$} & $15 \mathrm{~min} / 0.016 \mathrm{HAU}^{3}(\mathrm{H} 5)$ & \multirow{2}{*}{ Human serum } & \multirow{2}{*}{ [122] } \\
\hline $\begin{array}{l}\text { InfluenzaA virus } \\
\text { subtypesH5 and H9 }\end{array}$ & & & 0.25 HAU (H9) & & \\
\hline
\end{tabular}

${ }^{1}$ DNA strand displacement amplification; ${ }^{2}$ International units; ${ }^{3}$ Hemagglutinating units.

As stated above, one of the main limitations of fluorescence-based LFAs is the need of expensive and bulky fluorescence readers for quantitative analysis. Most of the reported fluorescence based LFAs use portable strip readers not suitable for POC applications (Fig. 3c). Even being portable, such fluorescence readers are still bulky and can only operate for few hours without a power supply. Less reported but remarkable examples of handheld devices [122,123,125,129] and smartphone-based fluoresce readers [127,130] can also be found in literature. 
a)
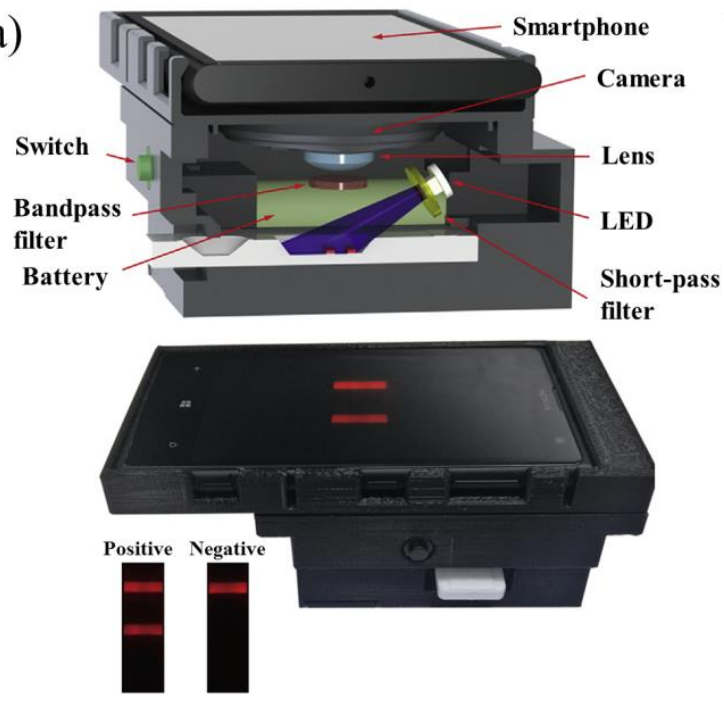

b)

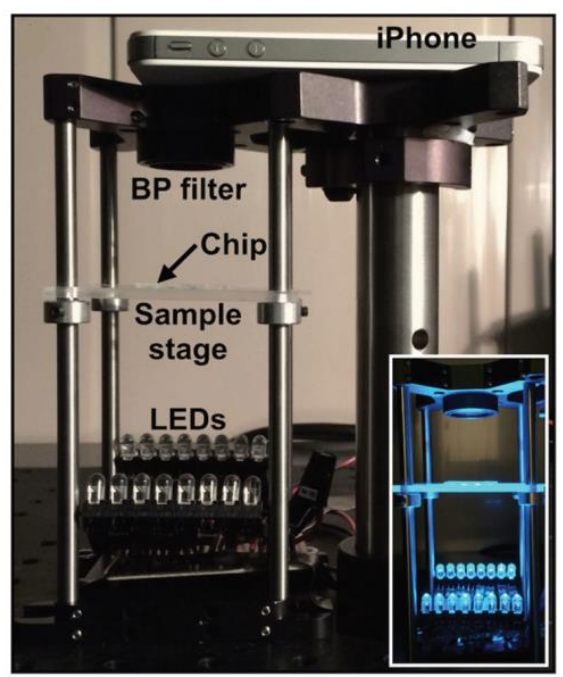

c)

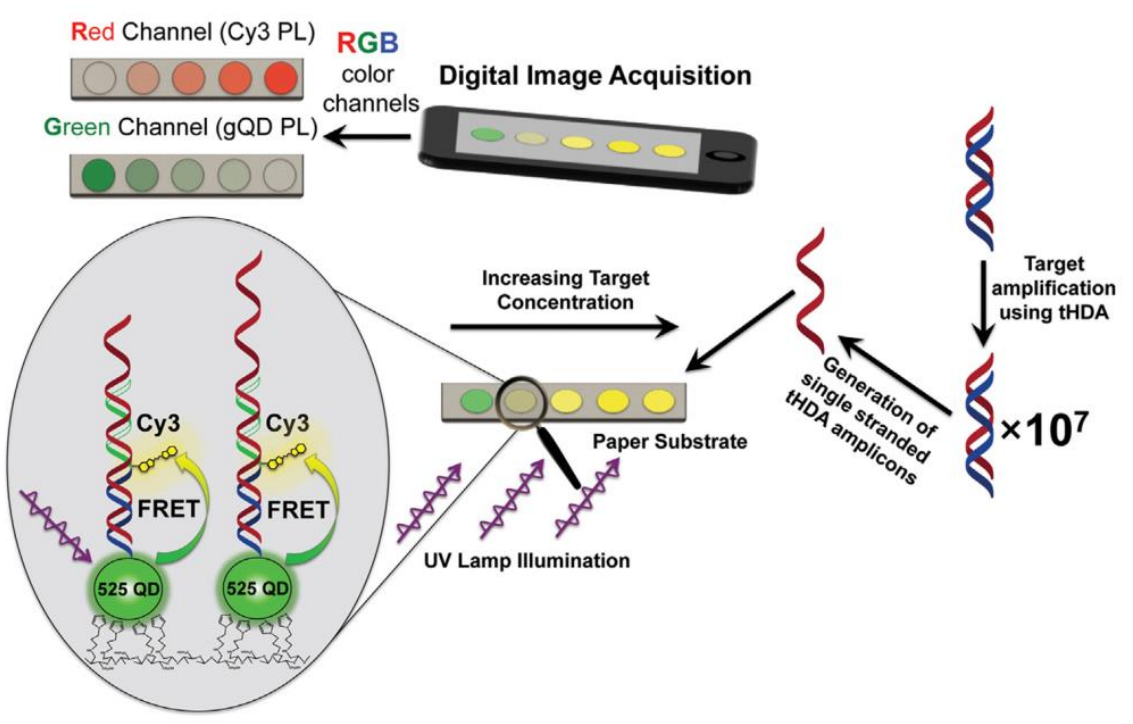

Figure 13. Smartphone-based QD biosensing systems. a) 3D schematic and photograph of the smartphone-based fluorescence LFAs reader developed for Zika detection (Reprinted with permission from [132]. Copyright 2019 Elsevier); b) Photograph of the setup used for smartphone readout of QD-FRET test strip for thrombin activity (Reprinted with permission from [134]. Copyright $2015 \mathrm{RSC}$ ); c) Schematic illustration of the ratiometric QD-FRET assay for DNA diagnostic of spinal muscular atrophy disorder and E. coli (Reprinted with permission from [129]. Copyright 2018 American Chemical Society). 
Smartphone-based platforms have also been used as detectors in QD-based FRET assays on paper substrates. FRET offers the possibility of a ratiometric quantification approach, which is able to correct for environmental factors and to self-calibrate. Ratiometric fluorescent intensities can also be easily monitored using a smartphone by simple splitting of red, green, and blue channels in a captured image.

In this context, Petryayeva et al. [132] reported the use of a smartphone detector of FRETbased paper test strips for thrombin activity in serum and whole blood using $\mathrm{CdSe} / \mathrm{CdS} / \mathrm{ZnS}$ as donors and Alexa Fluor 647 (A647) as acceptor (Figure 13b). Immobilized QDs, conjugated with an A647-labeled peptide substrate, respond to thrombin activity through the loss of FRET between the QD and A647 with recovery of quenched QD photoluminescence. Quantitative results were obtained in less than 30 min with a LOD of $18 \mathrm{NIH}$ units $\mathrm{mL}^{-1}$ of activity in whole blood.

Following a similar strategy, FRET-based assays for the quantification of a protein biomarker of epithelial tumors [133] and the DNA diagnostic of spinal muscular atrophy disorder and E. coli. [134] have been reported (Figure 13c). CdSSe/ZnS donors were used in combination with $\mathrm{Cy} 3$ fluorescent dye acceptor for a sensitive and selective detection of both, the epithelial cell adhesion protein and the oligo sequences. As in the $\mu$ PADs, the hydrophilic areas where QDs were immobilized on these paper-based assays were defined by wax patterning.

Although optical sensing has been by far the dominant detection method in the field of paper microfluidics, examples of electrochemical SQD-based paper devices for clinical diagnostics can also be found in literature [135,136]. Electrochemical sensing offers an alternative detection system for quantitative analysis in paper devices due to its simplicity, portability, and sensitivity. Small size electrodes can be easily integrated into the paper strip (Figure 14a) or fabricated directly onto the paper substrate (Figure 14b). These devices combined with commercially available miniaturized potentiostats appear as a very interesting alternative to fluorescence-based paper devices. 


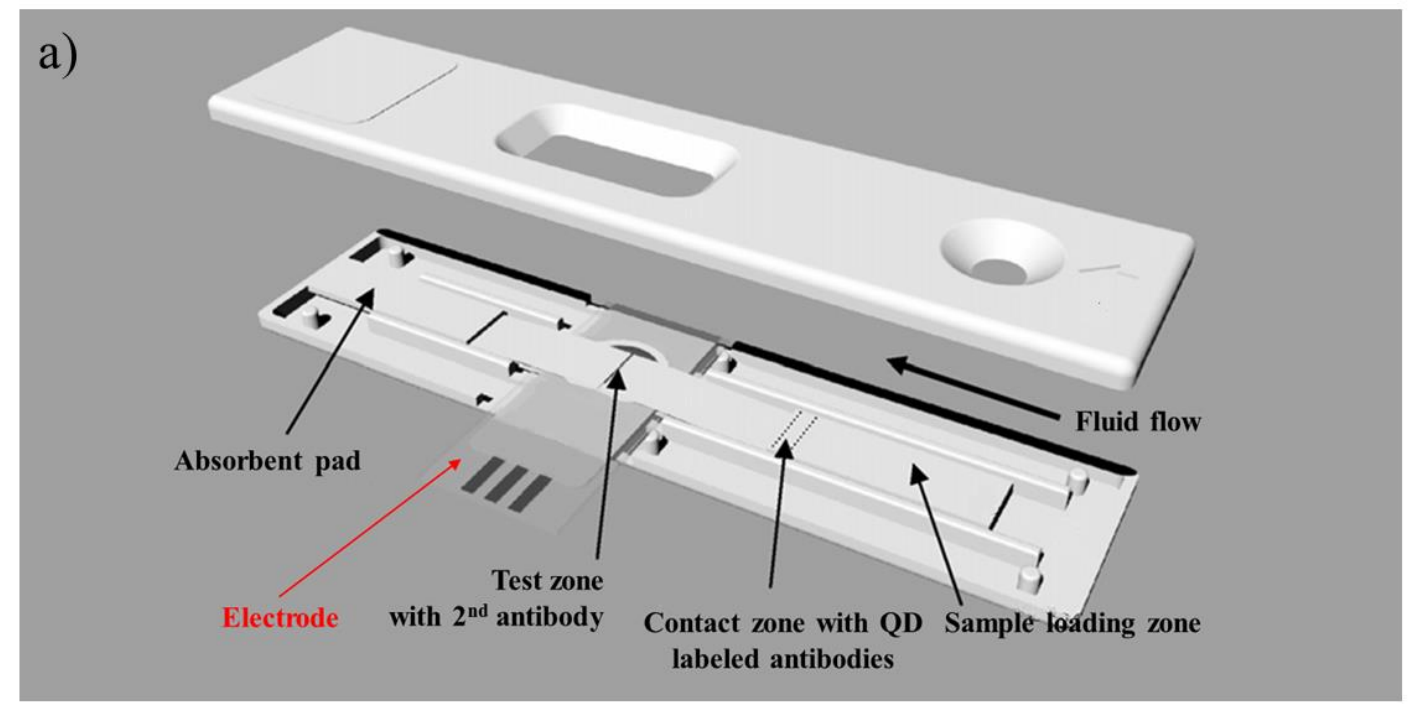

b)

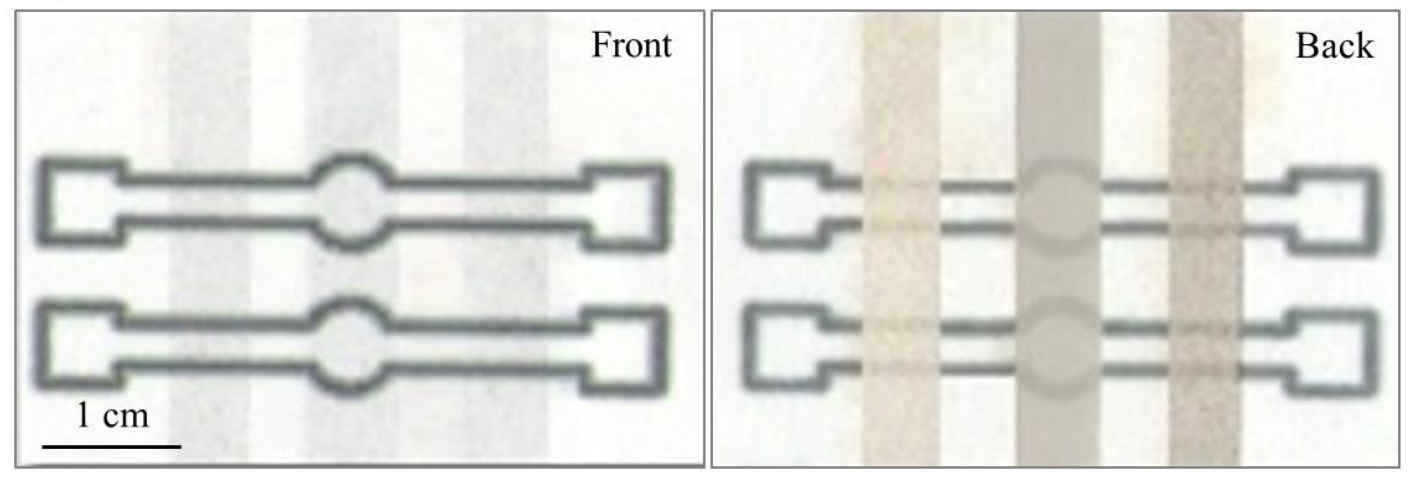

Figure 14. Electrochemical QD-based paper devices (Reprinted with permission from [136]. Copyright 2015 Elsevier). a) Schematic diagram of an electrochemical LFAs that integrates a screenprinted electrode underneath the test zone of the strip for QD detection; b) Photographs of the front and back face of an electrochemical paper devices with the electrodes deposited directly onto the paper substrate. Microfluidic pattern is wax-printed on the front face of the paper and the electrodes are deposited by sputtering on the back side of the paper.

\subsection{QD barcode POC systems}

Barcode assays for POC applications are highly demanded by modern healthcare systems [137]. Barcode assays are capable to simultaneously detect multiple targets from patient samples, thereby increasing the speed of analysis and improving the precision and accuracy of diagnosis. As mentioned above, QDs can be used as barcoded probes by embedding them 
into polymeric particles [97]. In this line, Gao et al. [138] reported a simple strategy to automate barcode assays in order to make them suitable for POC applications. A microfluidic device was designed to perform all steps of the assay using barcoded polystyrene microbeads consisting of magnetic NPs (FeO) and quantum dots ( $\mathrm{ZnS}$-capped $\mathrm{CdSeS}$ ). An on-chip sandwich hybridization assay for the detection of genetic targets for HIV, hepatitis B, and syphilis was successfully performed in only 20 min with a LOD of $1.2 \mathrm{nM}$. The POC application of this microfluidic device is, however, hindered due to the bulky instrumentation required for the fluorescence detection.

A smartphone reader combined with quantum dot barcoding technology was used by Ming et al [139] in the development of a low-cost chip-based wireless multiplex diagnostic device (Figure 15). Quantum dot barcodes were prepared using different rations of eight different SQDs embedded into a polymeric matrix. These quantum dot barcodes were then arrayed on microfabricated glass slides to create a multiplex chip platform simple to use and easy to transport. This handheld device (Figure 15) was capable of detecting down to 1000 viral genetic copies per milliliter enabling the diagnosis of patients infected with HIV or hepatitis $\mathrm{B}$ in less than $1 \mathrm{~h}$.

a)
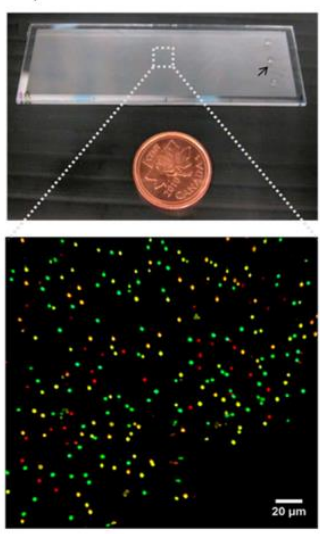

b)

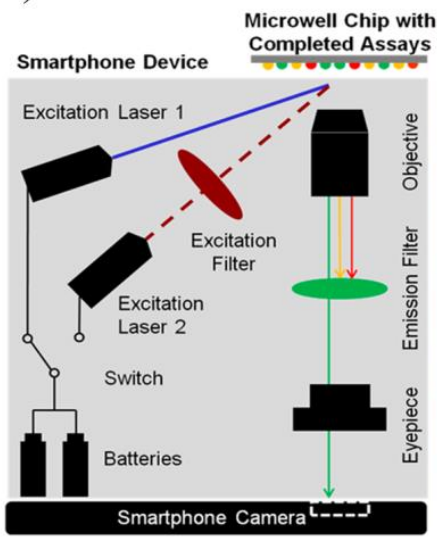

c)

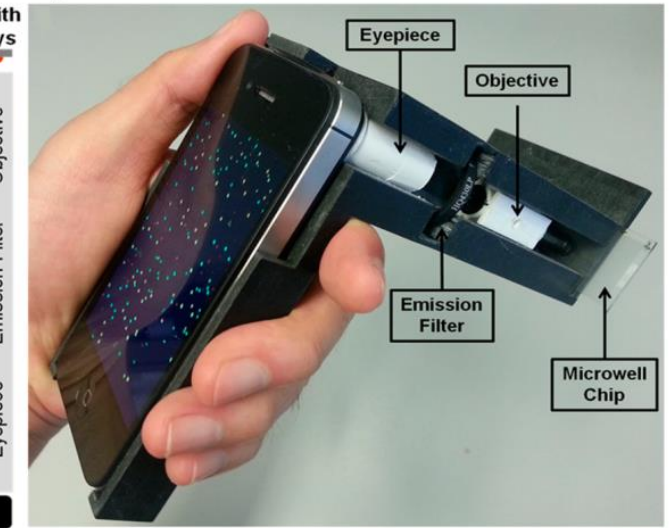

Figure 15. Overview of the smartphone device utilizing quantum dot barcodes :a) Photograph of microwell chip containing different barcodes in each well and image of four different quantum dot barcodes arrayed on the surface of the chip captured by a smartphone; b) Schematic illustration of the detection system performance; c) Image of the smartphone device. Reprinted with permission from [139]. Copyright 2015 American Chemical Society. 


\section{BIOMEDICAL LABELING AND IMAGING}

Functionalization of QDs enables them to play a major role in the field of diagnosis and medicine improving the capabilities of molecular imaging techniques. In molecular imaging, quality functional images (e.g. with high contrast to allow proper differentiation) are required. Among the different available techniques, fluorescence imaging is a powerful tool to effectively image eventual interactions occurring at the molecular level directly, at real time and with rather high sensitivity.

Unfortunately, conventional fluorescent dyes typically used as contrast agents in imaging techniques suffer of a poor tissue specificity, low stability (when entering in the biological media) and photobleaching problems, and reduced signal penetration needed for optimal performance. Alternatively, QDs appear as an exciting class of fluorescent probes for photoluminescence imaging because of their tunable optical properties, high stability, and the ability to be used in guided-targeting based on the NP surface functionalization with appropriate recognition elements (e.g. antibodies, aptamers, peptides, etc.). Researches have explored various methods based and QDs functionalization to enhance fluorescence imaging. This section includes a brief overview of the emerging applications for bioconjugated QDs in bioimaging for clinical diagnosis.

\subsection{Bioconjugated QDs for biomedical labeling and imaging.}

At present, increasing research attention is given to luminescence-based imaging of biological specimens to be used in biomedical studies, and of particularly importance in medical diagnostic, as developing technological advances point to enhanced biomedical capabilities (e.g. innovative fluorescence-based imaging systems). Typical fluorescent imaging reagents can be endogenous (that often require an enzyme-mediated process inside the organisms to stimulate the production of measurable visible light) or exogenous (that are today the more versatile and popular fluorescent imaging agents). NPs-based optical contrast agents (including QDs) are within the group of exogenous reagents that still are dominated today by sensitive organic fluorescent probes. However, the important advances on bioconjugation of QDs to selected recognition elements (e.g. antibodies, peptides, genetic material, etc.), constitutes a significant trust in the development of novel and improved 
bioimaging methodologies [140]. The produced QDs-biomolecule bioconjugates constitute outstanding nanoplatforms for fluorescence labeling of target biomolecules. Such fluorescent labels have been successfully employed in fluorescence imaging in the study of living cells single-molecule dynamics, monitoring of intracellular protein-protein interactions, disease detection in deeper tissues, detection of tumor cells based on selective binding of the tailored, bioconjugated QDs to known cancer biomarkers, and much more [141].

Pioneer studies of the use of QDs as luminescence tags for imaging exploited the high sensitivity of the luminescence from this NPs to the surface state. Eventual chemical or physical interactions between chemical species present in the media and the surface of the QDs would result in detectable changes on the fluorescence emission. Based on such basic approach, mercaptoacetic acid (MAA)-capped CdSe/ZnSe/ZnS QDs were evaluated for the detection of changes of $\mathrm{pH}$ within SKOV-3 human ovarian cancer cells. In this study, authors make use of the changes on the intrinsic fluorescence emission of QDs with the $\mathrm{pH}$, being the more intense fluorescence emission obtained at higher pHs. Therefore, after QDs were uptaken by endocytosis within the lysosomes, in both fixed and living cells, where the $\mathrm{pH}$ is rather low, addition of chloroquine produced an increase on the $\mathrm{pH}$ and an enhancement of the photoluminescence intensity of the QDs [142].

However, it must be taken into account that such methods based on direct interaction of the analytes with the QDs suffer from poor selectivity and so they have a rather limited applicability in real life. However, advances in controlled bioconjugation of the QDs to active recognition elements explains the myriad of applications of QDs recently emerging in targetguided fluorescence imaging. Selective detection of multiple tumor biomarkers, monitoring of molecular surface dynamics of membrane-associated molecules, measurement of cell motility or quantification of molecular interactions on the cellular and subcellular level through fluorescent imaging are just some few examples illustrating the present growing importance of the field [143].

For instance, the prominent relevance of cancer research in the life sciences has promote studies of location and distribution of tumors and tumour cells using fluorescent in vivo imaging. To get an insight in the trafficking of cancer cells, it is possible to label such cells with QDs surface modified with antibodies. As an example, QDs were encapsulated in 
carboxylated triblock polymeric micelles and conjugated with anti-mesothelin antibodies to allow targeting of cancerous areas [144]. An in vivo imaging study demonstrated that the QDs-loaded nanomicelles, surface modified with the antibodies, targeted the pancreatic tumor site only $15 \mathrm{~min}$ after intravenous injection, thus providing the potential of these structures as promising nanoscale platforms for early human pancreatic cancer detection.

When designing imaging contrast agents based on QDs the first challenge is to delivery successfully the nanoplatform into the cell. Cellular penetration of QDs can be achieved using different approaches based on active and passive transportation. The major transport pathway of QDs into cells is endocytosis, in which eukaryotic cells ingest a part of their plasma membrane to swallow external objects. Passive transduction of QDs into the cell is mostly enabled via electrostatic interaction with the plasma membrane. This is the mechanism more probable in the case of water-stabilized QDs without functionalization with biomolecules. However, in this scenario there is not a guarantee of efficient uptake.

It must be mentioned that up to date mechanism of cellular uptake and cytotoxicity of nanoparticles are still largely understood. Some studies demonstrated that cellular uptake pathways are strongly dependent on cell type and cell differentiation (e.g. Monocytes showed cellular uptake of QDs surface modified with carboxylic acid, while lymphocytes did not) [145]. Other studies demonstrated that QDs coatings highly affect their cellular uptake. As an example, polyetilenglycol surface-modification of QDs blocked non-specific QD delivery into the cells, while QDs coated with carboxyl or amine groups can be internalized quickly and with large amount by different types of cells [146]. Thus, it is difficult to provide a general conclusion about mechanisms for QDs cell uptake. In any case, in the report by Xiao, Y. et al [146], results obtained suggested a potential pathway for QD cellular uptake mechanism consisting on three main stages: (1) endocytosis that may occur through two major mechanisms named phagocytosis and pinocytosis. In the case of small QDs it is envisaged that endocytosis happens via micropinocytosis (a subcategory of pinocytosis that is preferred for the uptake of smaller particles through the formation of endocytic vesicles of different sizes) (2) sequestering in early endosomes; (3) translocation to later endosomes or lysosomes. Authors of the study found that endocytosis was probably assisted by receptors specific to ligands with negative charges. All these findings are of great relevance to improve 
specific targeting of QDs in bioanalytical and medical applications as it can be considered to reduce non-specific targeting.

Conversely, QDs active transportation is characterized by ligand-receptor mediated transportation using ligands, such as peptides, proteins or antibodies. In addition, QD surface engineering is critical to minimize undesired nonspecific binding adsorptions of QD probes in biological media. Surface-functionalization of QDs with uncharged hydrophilic moieties (e.g. PEG) or with zwitterion molecules produces highly water-stable nanoprobes while efficiently eliminating nonspecific binding (typically brought about by hydrophobic and/or pure electrostatic interactions).

The use of antibody-QD conjugates for guided-target labeling is probably the most typical approach in molecular imaging, although targeting can also be achieved via bioconjugation of the QDs with peptides, DNA, modified proteins, etc. Actually, a single QD (depending on its size) can be simultaneously conjugated with several proteins and peptides [147]. It is well known that peptides are efficient carriers of QDs inside living cells. Therefore peptide-modified QDs conjugates were widely used to target cellular biomolecules including growth factor receptors, G protein-coupled receptors, integrins and even ion channels [148].

Moreover, small biomolecules can be also bioconjugated to QDs for in vitro imaging. This is the case of aptamers, that are nucleic acid species that have been engineered to bind to various molecular targets such as small molecules, proteins, nucleic acids, and even cells, tissues and organisms [149]. As an illustration, $\mathrm{ZnS} / \mathrm{CdSe}$ fluorescent quantum dots were surface modified with PEG (for aqueous stabilization and biocompatibility) and streptavidine (to further bioconjugate the NP to an appropriate biomolecule receptor). The streptavidinePEG-QD was then labeled via streptavidine-biotine with a biotinylated aptamer which can specially bind the epidermal growth factor receptor (EGFRvIII) specially distributed on the surface of glioma cells. The so-prepared labeled aptamer (QD-Apt) nanoprobe was then employed in a fluorescence-guided surgery to allow a safe resection of glioma [150]. In this study, it was evaluated the biodistribution of the QD-Apt nanoprobe and the capabilities of targeted imaging of glioma in situ using orthotopic glioma models. Different studies were carried out, including an evaluation of the capability of the QD-Apt to image the U87EGFRvIII tumor areas. For such purpose, at 4 weeks of tumor development, a whole-body 
fluorescence imaging was performed in different mice after 6h tail vein injection of QDs (not bioconjugated) or QD-Apt. As can be seen in Figure 16, the image of the mice harboring tumor administered with QD-Apt (group 1) had strong fluorescence signals in tumor areas, while the group administered with QDs (group 2) had no obvious fluorescence signals in the tumor region. In addition, the mice bearing U87 tumors (groups 3 and 4) had no significant fluorescence in tumor areas, regardless of whether they were administered with QD-Apt or QDs. In brief, the developed nanoprobe (QD-Apt) have a great potential as a novel fluorescence contrast agent for the molecular diagnosis, image-guided surgery, and postoperative examination of gliomas.
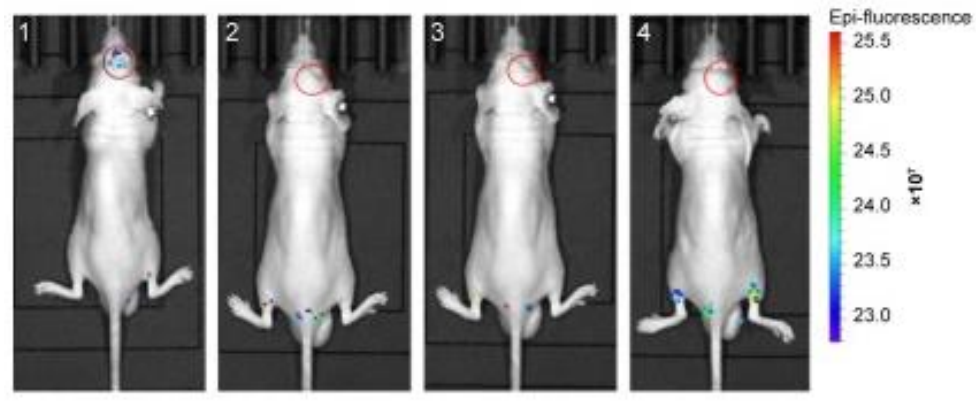

Figure 16. Effects of QD-Apt on tumor imaging and body distributions in vivo. Fluorescence images of the mice after injection of QDs or QD-Apt via tail vein for $6 \mathrm{~h}$. The red circles indicate the tumor regions. The mice of group 1 and group 2 were bearing U87-EGFRvIII tumors, while the mice of group 3 and group 4 were bearing U87 tumors. In addition, the mice of group 1 and group 3 were administered with QD-Apt, while the mice of group 2 and group 4 were administered with QDs. The scale bar values are $100 \mu \mathrm{m}$. All pictures are magnified 200×. Reprinted with permission from [150]. Copyright 2018 Wiley.

Clearly, engineering more compact nanoprobes for fluorescence imaging is today a research area of great practical interest. A key aspect in this task is try to enhance the final uptake of the QDs-based nanoprobes in the cellular media. For such purpose, much an increase amount of work is being carried out in order to avoid (or reduce) eventual QDs aggregation or deposition in endosomes or lysosomes. In addition, studies to achieve an efficient QD surface engineering are critical to minimize undesired nonspecific binding 
problems of QD nanoprobes when entering the biological media (a problem limiting the applicability of these nanostructures).

\subsection{Near-Infrared Optical Imaging.}

Fluorescent QDs with emission within the visible spectral range are mostly limited to in vitro bioimaging applications, because tissues have strong absorbance in the visible region. Additionally, endogenous autofluorescence in the visible spectrum from biological components present in living tissues may significantly interfere with signals from QDlabeled biomolecules. Alternatively, recently there is a high interest in the preparation of nanoprobes made of QDs with emission in the near-infrared (NIR) spectral region to overcome such limitations. NIR light is able to pass more efficiently through biological tissues, suffering from much lower absorption and minimum biological autofluorescence, making QDs with emission in the NIR range highly attractive to maintain their high image resolution even when labeling deeper tissues in vivo [151]. As a result, NIR QDs are more attractive than visible-emitting QDs for in vivo mapping and imaging applications as the use of NIR emission substantially increases contrast, sensitivity, and penetration depth while avoiding optical damage to the body [152].

As an example, Bawendi and colleagues bioconjugated NIR InAs(ZnCdS) QDs with a polymeric imidazole ligand via a ligand exchange strategy obtaining nanoprobles with bright and stable emission in the near-infrared (NIR, 700-900 $\mathrm{nm}$ ) region. They evaluated the potential of such nanoprobe to image tumor vasculature, in vivo [153]. As can be seen in Figure 17, authors demonstrated that the NIR-emitting QDs offered superior depth and contrast when compared to green QDs. The NIR QDs clearly image deep vasculature of a mammary cell with an excellent resolution up to $200 \mu \mathrm{m}$, while the visible emitting QDs produce an image with much lower contrast and emission intensity. 


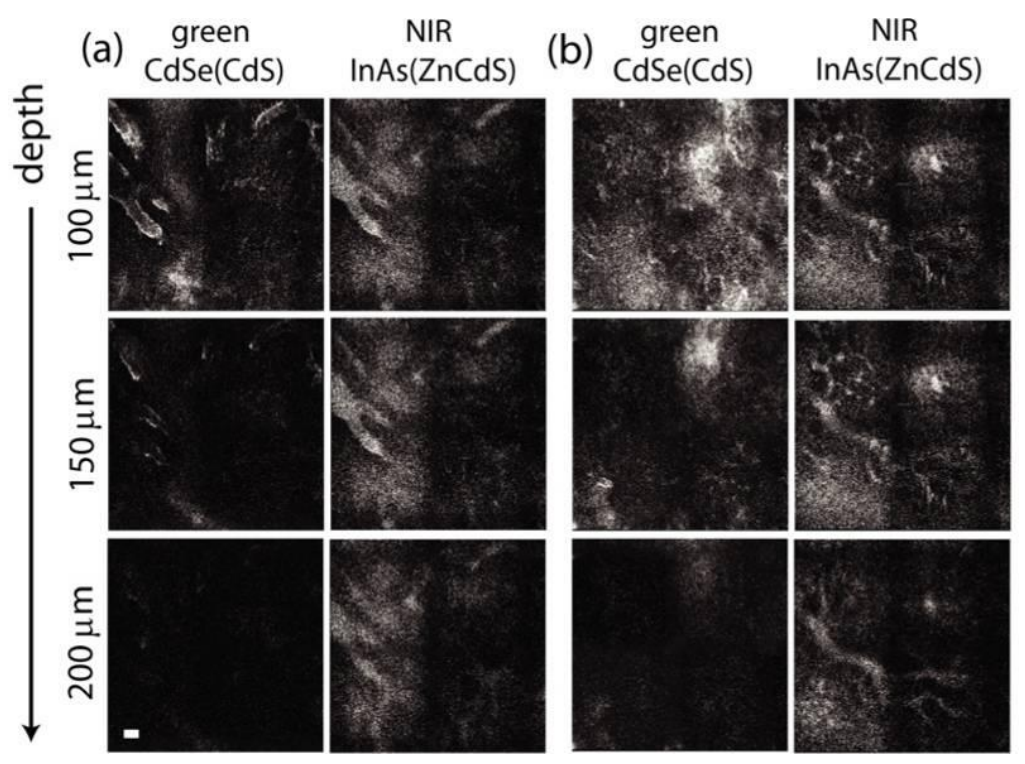

Figure 17. In vivo grayscale MPM images with $850 \mathrm{~nm}$ excitation (a-b) of the vasculature in a mammary tumor in a mouse with $\mathrm{CdSe}(\mathrm{CdS})$ (green channel) and $\mathrm{InAs}(\mathrm{ZnCdS})$ (NIR channel) poly(PEG12)-PIL QDs injected intravenously and imaged simultaneously (scale bar, $100 \mu \mathrm{m}$ ). Reprinted with permission from: Allen PM, Liu W, Chauhan VP, Lee J, Ting AY, Fukumura D, Jain RK, Bawendi MG (2010) InAs(ZnCdS) Quantum Dots Optimized for Biological Imaging in the NearInfrared. J Am Chem Soc 132:470-47. Copyright 2010 American Chemical Society.

Two of the most robust NIR-emitting QD are the ternary CuInS2 and CuInSe 2 core-shell systems. Quite recently, CuInSe2/ZnS QDs with a NIR emission (709 nm) were synthesized and surface conjugated with the tumor targeting peptide Cys-Gly-Lys-Arg-Lys (CGKRK) through a PEG linker [154]. It was observed that QDs functionalized with the peptide increased the NP tumor uptake by over two times as compared to QDs functionalized with PEG alone. The bioconjugated QDs allowed real time imaging of the tumor homing process in live mice for a long period of time. The functionalized QDs showed high photostability and were cleared fairly slowly (half-life of $\approx 7 \mathrm{~h}$ ).

Imaging experiments carried out in vivo have demonstrated that an improvement in the overall signal-to-noise ratio over a 100-fold was found when employing as contrast agents NIR QDs emitting at $1320 \mathrm{~nm}$ (NIR-II region) rather than those QDs emitting at $850 \mathrm{~nm}$ (NIR-I region), as a consequence of a reduced autofluorescence along with a superior tissue penetration observed at those larger wavelengths. Thus, recently some efforts have been done 
trying to develop QDs that emit in the NIR-II window based on NP cores of different metallic elements such as $\mathrm{PbSe}, \mathrm{PbS}$ or hybrid CdHgTe. More recently, NIR-II QDs have also been synthesized avoiding the use of toxic elements: a mirage of reports propose $\operatorname{Ag}_{2} \mathrm{~S}$ QDs as NIR-II emitting agents.

Up to date, only few reports deal with successful specific targeting employing $\mathrm{Ag}_{2} \mathrm{~S}$ QDs [155]. In all, $\mathrm{Ag}_{2} \mathrm{~S}$ NPs were surface-functionalized with appropriate recognition elements for in vivo imaging following different bioconjugation approaches. In a very recent study $\mathrm{Ag}_{2} \mathrm{~S}$ NPs were surface functionalized with plerixafor (a small molecule drug used for inhibition of a CXC chemokine receptor 4, CXCR4). The bioconjugate was used for in vivo imaging of metastatic breast cancer cells based on the selective linkage of the functionalized $\mathrm{Ag}_{2} \mathrm{~S}$ NPs to highly metastatic breast cancer cells (4T1 tumor model) via their CXCR4 receptor [156]. Moreover, the use of the surface-decorated $\mathrm{Ag}_{2} \mathrm{~S}$ NPs together with their photothermal properties resulted in a unique and tumor-specific theranostic element (see Figures $18 \mathrm{a}$ and $18 \mathrm{~b})$.

(a)

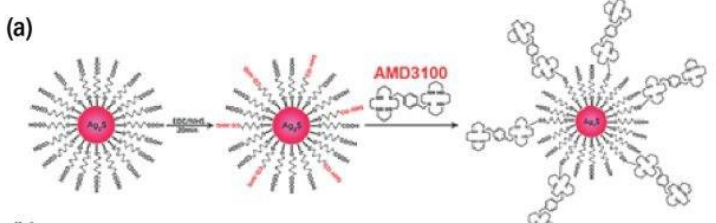

(b)
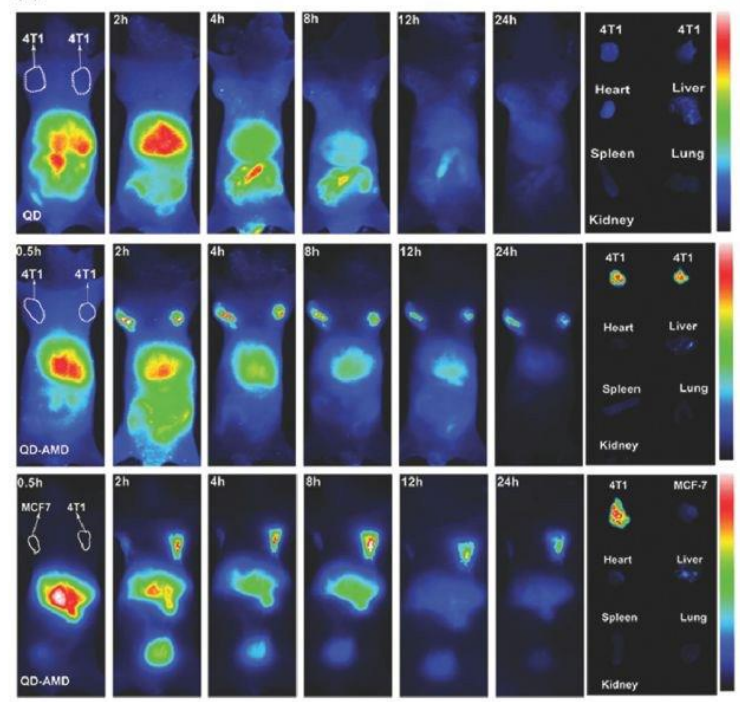

(c)

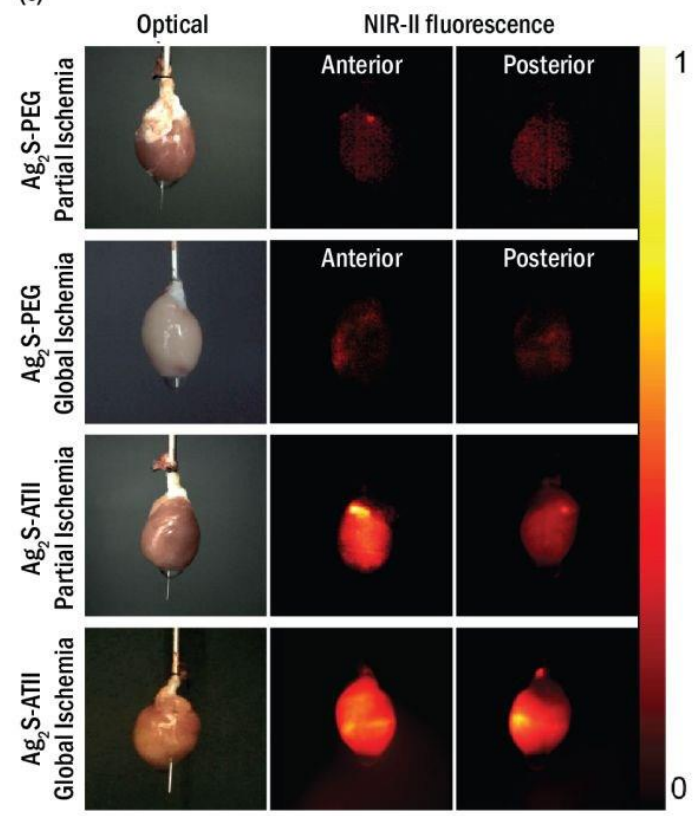

Figure 18. a) Schematic representation of $\mathrm{Ag}_{2} \mathrm{~S}$ NPs surface-functionalized with plerixaflor (QDAMD). b) Top-row: Time distribution in mice bearing bilateral 4T1 tumors employing non- 
targeting $\mathrm{Ag}_{2} \mathrm{~S}$ NPs and fluorescence of the excised organs, evidencing non-selective accumulation. Middle row: in vivo imaging in mice bearing bilateral 4T1 tumors with QD-AMD showing accumulation in the tumors. Bottom row: in vivo imaging in mice bearing MCF-7 and 4T1 tumors, respectively, at two axilla sites using QD-AMD resulting in the selective marking of 4T1. c) Optical and fluorescence images of the infarcted (anterior) and the opposite (posterior) side of rat hearts exposed to 60 min partial and global ischemia. The non-specific $\mathrm{Ag}_{2} \mathrm{~S}-\mathrm{PEG}$ NPs result only in weak fluorescence (top 2 rows), while the functionalized $\mathrm{Ag}_{2} \mathrm{~S}$-ATII NPs demonstrate their targeting of infarcted tissues, illustrated by the partial ischemia ( 3 rd row) where only one side of the heart is affected and the global event, where damaged tissue is identified on both sides (4th row). Adapted with permission from references [156] and [157]. Copyright 2018 WILEY-VCH Verlag GmbH \& Co. KGaA, Weinheim and Tsinghua University Press and Springer-Verlag GmbH Germany, part of Springer Nature 2019. 


\subsection{Multimodal imaging.}

Today there is an increasing research interest focused in the development of new nanomaterials for multimodal imaging applications in biology and medicine. Particularly, multimodal fluorescent-magnetic based nanomaterials deserve particular attention as they can be used both as diagnostic and drug delivery tools, which could facilitate the diagnosis and treatment of many diseases.

In this context, probably the most frequently investigated QD-based hybrid-NPs with multiple capabilities are magnetic-QDs. As an example, different sized infrared-emitting QDs have been incorporated, along with variable amounts of Fe-based magnetic NPs, into poly(styrene/acrylamide) copolymer nanospheres for the preparation of fluorescent-magnetic nanocomposites [158]. The dual-encoded nanobioprobes here developed, exhibited different luminescent behavior (because of the different sizes of the NPs) as well as magnetic susceptibility. They were proven capable of simultaneously recognizing and separating multiple biocomponents from complex samples when three kinds of lectins were used as the targets.

Multimodal imaging can integrate structural/functional information from several imaging modalities, thus promising more accurate diagnosis than any single imaging modality. One important advantageous feature of liposomes encapsulation is the possibility to coimmobilize several NPs exhibiting different properties to develop multimodal imaging platforms. In a very recent article, a theranostic liposome (QSC-Lip) integrated with superparamagnetic iron oxide NPs (SPIONs) and quantum dots (QDs) and cilengitide (CGT) into one platform is constructed to target glioma under magnetic targeting (MT) for guiding surgical resection of glioma [159]. In vivo dual-imaging studies show that QSC-Lip not only produces an obvious negative-contrast enhancement effect on glioma by magnetic resonance imaging but also makes tumor emitting fluorescence under MT.

Among the different techniques widely used for molecular imaging, today magnetic resonance imaging is among one of the main in vivo imaging techniques used routinely in diagnosis, while fluorescence imaging is nowadays most widely used for in vitro studies, making those imaging techniques complementary. Clearly, there is a high research interest in preparing fuorescent imaging/magnetic resonance imaging dual-modality nanoprobes to 
be used in many diagnostic and biomedical applications such as in cell labeling, enzyme activity measurements, tumor diagnosis and therapy, and anatomical localization and realtime assessment during surgery [160]. Nanostructures based on fluorescent QDs can be synthesized providing magnetic properties to the nanomaterial offering opportunities for multi-modality biomedical imaging. Fluorescent QDs exhibiting magnetic susceptibility can be synthesized following four different methodologies: metal doping, covalent conjugation, isocrystal growth and co-encapsulation or electrostatic assembly. Particularly, a large number of FI/MRI dual-modality imaging nanoprobes combine $\mathrm{Gd}^{3+}$ or $\mathrm{Mn}^{2+}$ ions with QDs. As an example, a dual contrast nanoreagent was developed by doping gadolinium ions into $\mathrm{CuInS}_{2} / \mathrm{ZnS}$ QDs (see Figure 19) [161]. The resulting NPs exhibited near-infrared fluorescence emission and MRI contrast capabilities with a high longitudinal relaxivity (r1), which was 2.5 times as high as that of clinically approved Gd agents. In addition, the in vivo imaging experiments showed that the Gd-doped NPs could enhance both near-infrared fluorescence and T1-weighted magnetic resonance (MR) imaging of tumor tissue through passive targeting accumulation.

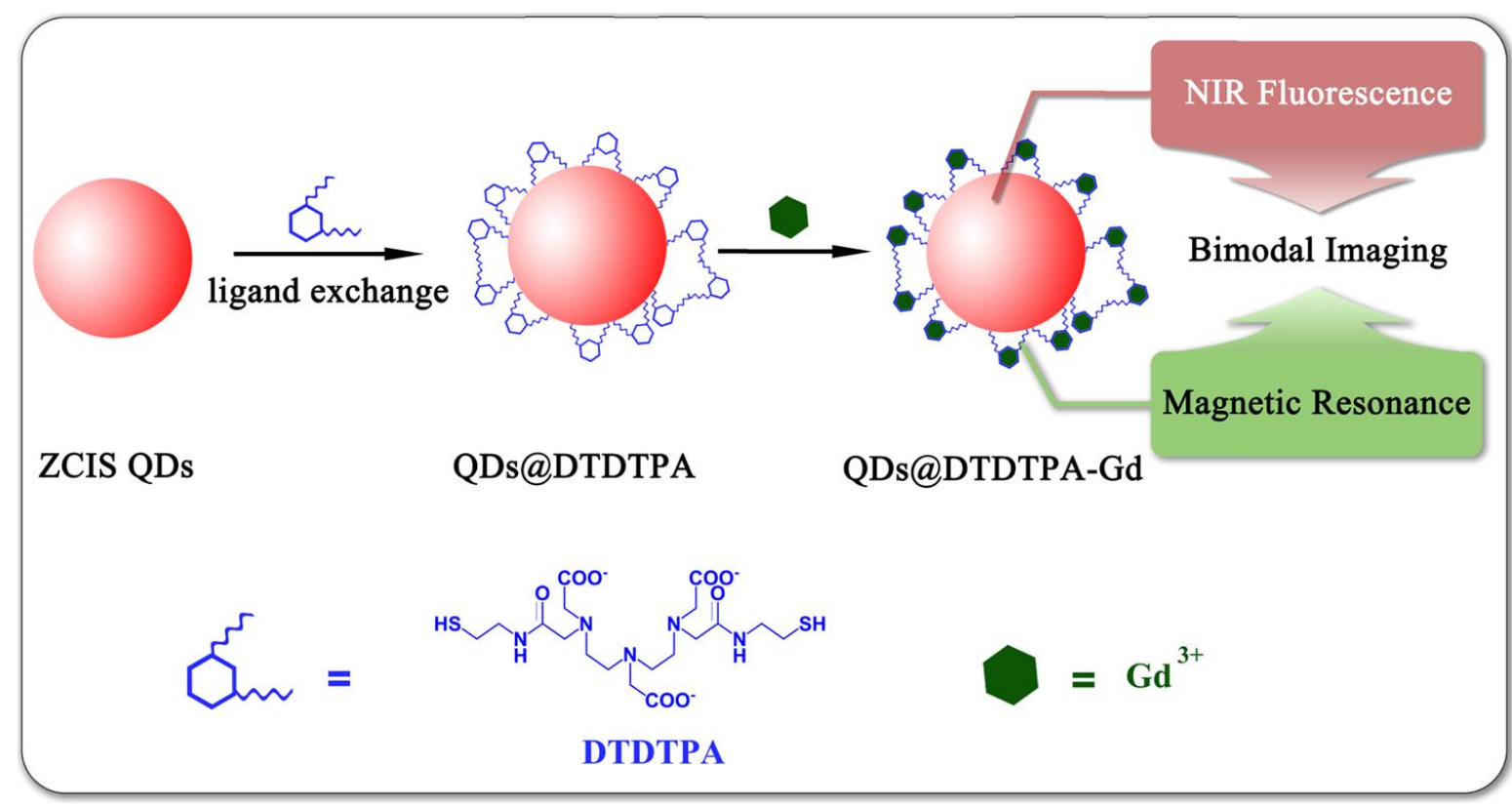

Figure 19. Fabrication procedure and functional description of the Gd-doped QDs with dualmode imaging capabilities. Reprinted with permission from Ref. [161]. Copyright 2017 American Chemical Society. 


\section{CONCLUSIONS AND PERSPECTIVES}

In general, photoluminescent quantum dots probes have been found widespread and are used in countless biomedical applications. For biosensing, a great potential of QD-conjugates also lies in multiplexing as well as in vitro and in vivo fluorescent imaging. In vivo drug delivery have also made a lot of progress and attracted great interest in this area. It is important to note that all of these applications are possible because of the advances on QDs stabilization in biological media by appropriate surface-functionalization and on their bioconjugation to appropriate biomolecules.

All recent advances in bioconjugation chemistry has made it possible to attach almost any biomolecule of interest to the QD surface. However, still there are some work to be done to further enhance the development and applications of QD bioconjugates. Particularly the following issues have been identified:

A key point that still needs to be appropriately addressed is the orientation of the biomolecules. Many often, random orientations are sufficient when the QDs are used in conventional hybridization applications. However, controlled orientations may be needed for assembly of functionalized 3D structures

Clearly, functionalization of QDs with different biomolecules (e.g., antibodies, peptides, nucleic acids or aptamers) offer a wide range of opportunities for applying the nanoassemblies in clinical diagnosis, including ultrasensitive detection of disease biomarkers, for in vivo targeted imaging or in drug delivery applications. The multiplexing capabilities of these QDs opens new avenues for the use of different-size QDs for simultaneous detection of multiple biomarkers (a key aspect in efficient clinical diagnosis). Moreover, encapsulation of multiple QDs in high-volume nanocarriers (e.g., nanosomes or PLGA NPs) may build a panel of multifunctional systems for targeted drug delivery and molecular imaging.

Recently, a great research interest is given to the development of new multimodal imaging nanoprobes. The use of NPs (NPs) as imaging probes offers several advantages over conventional molecular-scale contrast agents, such as high loading capacity, where the concentration of the imaging agents can be controlled within each NP during the synthesis process; tunable surface that can potentially extend the circulation time of the contrast agents 
in the blood, or target them to specific locations in the body; or provide multimodal imaging capacities because NPs can combine two or more contrast properties, which can be used in multiple imaging techniques simultaneously [162]. Recent advances in nanotechnology enabled the development of multifunctional QDs by doping the nanocrystals with appropriate metals, thus integrating two or more imaging contrast agents that allow their detection by different imaging techniques [42].

One of the major challenges when developing novel bioassay methods for clinical applications is the requirement of achieving high sensitivity in the detection, because of the ultralow concentrations of the biomarkers to be detected. Here, use of QDs as tags in immunoassays could be a powerful approach to achieve the desired ultrasensitivity. As an example, ultrahigh sensitivity for biomolecules could be easily achieved through metal deposition on the surface of the NP tags acting as catalytic seeds, thus effectively amplifying the size of the metallic NPs after the immunoassay [163].

Obviously, QDs cannot be safely used as tags for in vivo applications until the problem of their toxicity is solved. Despite very extensive studies of toxicity of QDs in different cellular and animal models the in vivo toxicological of effect of QDs still remains controversial [164]. It should be taken into account that extensive UV illumination may induce toxic heavy metal liberation from the QDs core [44]. Preparation of heavy metal-free QDs is being addressed as a promising avenue to overcome such toxicity problems. Additionally, it must be considered that an ideal solubilization strategy should reduce QD toxicity and undesirable nonspecific QD uptake by living tissues, thus reducing cytotoxic effects.

Paper-based microfluidic systems have been revealed as the most suitable platform for POC analysis, being the use of SQDs as labels becoming increasingly popular in the development of this type of systems. Although fluorescence SQD-based POC systems using hand-held readers or even smartphone based detectors have been successfully reported, they suffer of an important limitation related to the need of bulky and complex detectors for quantification. However, it is expected that the use of alternative detection methods (e.g. electrochemical) and the rapid development of portable devices and mobile phone technology will allow the miniaturization of the detection systems for POC devices in the near future. Miniaturized signal-recording devices also requires to merge QD barcode 
technology with POC testing. In the sensing field, there is also a great expectation on recently discovered graphene and carbon QDs.

Even though there is still a long road for the routine use of bioconjugated QDs for in vitro and especially in vivo diagnosis, overall, we firmly believe that the rapid furtherdevelopment of new bioconjugated nanomaterials will move bioconjugated QDs forward to real-life diagnostic applications in modern biology and medicine.

\section{ACKNOWLEDGEMENTS}

Financial support from the FC-GRUPIN-ID/2018/000166 project (Asturias Regional Government, Spain) and the CTQ2017-86994-R and CTQ2016-79412-P projects (MINECO, Spain) is gratefully acknowledged. A. de la Escosura-Muñiz acknowledges the MICINN (Spain) for the "Ramón y Cajal” Research Fellow (RyC-2016-20299).

\section{REFERENCES}

[1] Prado M, Espiña B, Fernández-Argüelles MT, Diéguez L, Fuciños P, Vial S, Oliveira JM, Reis RL, Boehme K (2016) Detection of foodborne pathogens using nanoparticles. Advantages and trends. In: Barros-Velázquez J (Ed) Antimicrobial Food Packaging, Elsevier Inc.

[2] Trapiella-Alfonso L, Llano-Suárez P, Sanz-Medel A, Costa-Fernández JM, FernándezArgüelles MT (2017) Analytical Nanoscience and Nanotechnology. In: Encyclopedia of Analytical Chemistry, John Wiley \& Sons, Ltd.

[3] Lopez-Lorente A, Valcarcel M (2016) The third way in analytical nanoscience and nanotechnology: Involvement of nanotools and nanoanalytes in the same analytical process. Trends Anal Chem, 75: 1-9.

[4] Blanco-López MC, Rivas M (2019) Nanoparticles for bioanalysis. Anal Bioanal Chem, 411: 1789-1790.

[5] Ahsan MA, Jabbari V, Imam MA, Castro E, Kim H, Curry ML, Valles-Rosales DJ, Noveron JC (2020) Nanoscale nickel metal organic framework decorated over graphene oxide and carbon nanotubes for water remediation, Sci Total Environ 698: 134214 
[6] Cova CM, Zuliani A, Santiago ARP, Caballero A, Muñoz-Batista MJ, Luque R (2018) Microwave-assisted preparation of $\mathrm{Ag} / \mathrm{Ag} 2 \mathrm{~S}$ carbon hybrid structures from pig bristles as efficient HER catalysts, J Mater Chem A 6: 21516-21523

[7] Ahsan MA, Deemer E, Fernandez-Delgado O, Wang H, Curry ML, El-Gendy AA, Noveron JC (2019) Fe nanoparticles encapsulated in MOF-derived carbon for the reduction of 4-nitrophenol and methyl orange in water, Catal Commun 130: 105753

[8] Ahsan MA, Jabbari V, El-Gendy AA, Curry ML, Noveron JC (2019) Ultrafast catalytic reduction of environmental pollutants in water via MOF-derived magnetic $\mathrm{Ni}$ and $\mathrm{Cu}$ nanoparticles encapsulated in porous carbon, App Surf Sci 497: 143608

[9] Ahsan MA, Fernandez-Delgado O, Deemer E, Wang H, El-Gendy AA, Curry ML, Noveron JC (2019) Carbonization of Co-BDC MOF results in magnetic C@Co nanoparticles that catalyze the reduction of methyl orange and 4-nitrophenol in water, J Mol Liq 290: 111059

[10] Sanad MF, Shalan AE, Bazid SM, Serea ESA, Hashem EM, Nabiha S, Ahsan MA (2019) A graphene gold nanocomposite-based 5-FU drug and the enhancement of the MCF7 cell line treatment, RSC Adv 9: 31021-31029

[11] Dominguez N, Torres B, Barrera LA, Rincon JE, Lin Y, Chianelli RR, Ahsan MA, Noveron JC (2018) Bimetallic CoMoS composite anchored to biocarbon fibers as a highcapacity anode for Li-ion batteries, ACS Omega 3: 10243-10249

[12] Piccinno F, Gottschalk F, Seeger S, Nowack B (2012) Industrial production quantities and uses of ten engineered nanomaterials in Europe and the world, J Nanopart Res 14: 1109 [13] Snee PT (2020) Semiconductor quantum dot FRET: Untangling energy transfer mechanisms in bioanalytical assays, Trends Anal Chem 123:115750

[14] Llano Suarez P, García-Cortés M, Fernández-Argüelles MT, Ruiz Encinar J, Valledor M, Ferrero FJ, Campo JC, Costa-Fernandez JM (2019) Functionalized phosphorescent nanoparticles in (bio)chemical sensing and imaging - A review, Anal Chim Acta, 1046: 1631.

[15] Granada-Ramírez DA, Arias-Cerón JS, Rodriguez-Fragoso P, Vázquez-Hernández F, Luna-Arias JP, Herrera-Perez JL, Mendoza-Álvarez JG (2018) Quantum dots for biomedical applications, Nanobiomaterials, 411-436 
[16] Coto-García AM, Sotelo-González E, Fernández-Argüelles MT, Pereiro R, CostaFernández JM, Sanz-Medel A (2011) Nanoparticles as fluorescent labels for optical imaging and sensing in genomics and proteomics, anal Bioanal Chem, 399: 29-42.

[17] Geißler D, Charbonnière LJ, Ziessel RF, Butlin NG, Löhmannsröben H-G, Hildebrandt N (2010) Quantum Dot Biosensors for Ultrasensitive Multiplexed Diagnostics. Angew Chem, 49: 1396-1401.

[18] Hildebrandt, N (2011) Biofunctional Quantum Dots: Controlled Conjugation for Multiplexed Biosensors. ACS Nano 5: 5286-5290

[19] Chan, W. C., Nie, S. (1998) Quantum dot bioconjugates for ultrasensitive nonisotopic detection. Science 281: 2016-2018.

[20] Sukhanova, A., Devy, J., Venteo, L., Kaplan, H., Artemyev, M., Oleinikov, V., Klinov, D., Pluot, M., Cohen, J. H., Nabiev, I. (2004) Biocompatible fluorescent nanocrystals for immunolabeling of membrane proteins and cells. Anal. Biochem. 324: 60-67.

[21] Gill R, Zayats M, Willner I (2008) Semiconductor Quantum Dots for Bioanalysis. Angew Chem Int Ed 47:7602-7625

[22] Bear J, Charron G, Fernandez-Arguelles MT, Massadeh S, McNaughter P, Nann, T (2011) In Vivo Applications of Inorganic Nanoparticles. In: Booss -Bavnbek B, Klosgen B, Larsen J, Pociot F, Renstrom E (ed) BetaSys. Systems Biology, vol 2. Springer, New York [23] Lu C, Chen G, Yu B, Cong H (2018) Recent Advances of Low Biological Toxicity Ag2S QDs for Biomedical Application. Adv. Eng. Mater., 20: 1700940.

[24] Rabani E (2001)Structure and electrostatic properties of passivated CdSe nanocrystals. J. Chem. Phys. 115:1493

[25] Jin Z, Hildebrandt N (2012) Semiconductor quantum dots for in vitro diagnostics and cellular imaging. Trends Biotechnol 30:394-403

[26] Alivisatos AP (1996) Perspectives on the Physical Chemistry of Semiconductor Nanocrystals. J Phys Chem 100:13226-13239

[27] Coto-Garcia AM, Fernandez-Arguelles MT, Costa-Fernandez JM, Sanz-Medel A, Valledor M, Campo JC, Ferrero FJ (2013) The influence of surface coating on the properties of water-soluble CdSe and CdSe/ZnS quantum dots. J Nanopart Res 15:1330

[28] Wagner MK, Li F, Li J, Li X, Le XC (2010) Use of quantum dots in the development of assays for cancer biomarkers. Anal Bioanal Chem 397: 3213-3224 
[29] Fernandez-Arguelles MT, Costa-Fernandez JM, Pereiro R, Sanz-Medel A (2010) Organically Modified Quantum Dots in Chemical and Biochemical Analysis. In: Rurack K, Martinez-Manez R (Ed) The Supramolecular Chemistry of Organic-Inorganic Hybrid Materials Wiley, New Jersey.

[30] Garcia-Cortes M, Sotelo E, Fernandez-Arguelles MT, Encinar JR, Costa-Fernandez JM, Sanz-Medel A (2017) Capping of Mn-doped ZnS quantum dots with DHLA for their stabilization in aqueous media: determination of the nanoparticle number concentration and surface ligand density. Langmuir 33:6333-6341

[31] Wu P, Yan XP (2013) Doped quantum dots for chemo/biosensing and bioimaging. Chem Soc Rev 42:5489-5521

[32] Panda SK, Hickey SG, Demir HV, Eychmüller A (2011) Bright white-light emitting manganese and copper co-doped ZnSe quantum dots. Angew Chem Int Ed 50:4432-4436 [33] Ehlert O, Osvet A, Batentschuk M, Winnacker A, Nann T (2006) Synthesis and spectroscopic investigations of $\mathrm{Cu}$ - and $\mathrm{Pb}$-doped colloidal $\mathrm{ZnS}$ nanocrystals. J Phys Chem B 110:23175-23178

[34] Sotelo-Gonzalez E, Fernandez-Arguelles MT, Costa-Fernandez JM, Sanz-Medel A (2012) Mn-doped ZnS quantum dots for the determination of acetone by phosphorescence attenuation. Anal Chim Acta 712: 120-126

[35] Mohapatra S, Rout SR, Das RK, Nayak S, Ghosh SK (2016) Highly hydrophilic luminescent magnetic mesoporous carbon nanospheres for controlled release of anticancer drug and multimodal imaging. Langmuir 32:1611-1620

[36] Wang J, Zhang Z, Zha S, Zhu Y, Wu P, Ehrenberg B, Chen JY (2014) Carbon nanodots featuring efficient FRET for two-photon photodynamic cancer therapy with a low fs laser power density. Biomaterials 35:9372-9381

[37] Chong Y, Ma Y, Shen H, Tu X, Zhou X, Xu J, Dai J, Fan S, Zhang Z (2014) The in vitro and in vivo toxicity of graphene quantum dots. Biomaterials 35:5041-5048 [38] Wang J, Zhang P, Huang C, Liu G, Leung K CF, Wáng YXJ (2015) High performance photoluminescent carbon dots for in vitro and in vivo bioimaging: effect of nitrogen doping ratios. Langmuir 31:8063-8073

[39] Vasimalai N, Vilas-Boas V, Gallo J, Cerqueira MF, Menéndez-Miranda M, CostaFernández JM, Diéguez L, Espiña B, Fernández-Argüelles MT (2018) Green synthesis of 
fluorescent carbon dots from spices for in vitro imaging and tumour cell growth inhibition. Beilstein J Nanotechnol 9:530-544

[40] Righetto M, Privitera A, Fortunati I, Mosconi D, Zerbetto M, Curri ML, Corricelli M, Moretto A, Agnoli S, Franco L, Bozio R, Ferrante C (2017) Spectroscopic Insights into carbon dot systems. J. Phys. Chem. Lett. 8:2236-2242

[41] Dong Y, Wang R, Li G, Chen C, Chi Y, Chen G (2012) Polyamine-functionalized carbon quantum dots as fluorescent probes for selective and sensitive detection of copper ions. Anal Chem 84:6220-6224

[42] Bouzas-Ramos D, Cigales-Canga J, Mayo JC, Sainz RM, Ruiz Encinar J, CostaFernandez JM (2019) Carbon Quantum Dots Codoped with Nitrogen and Lanthanides for Multimodal Imaging. Adv. Funct. Mater. 29: 1903884.

[43] Manna L, Scher EC, Li LS, Alivisatos AP (2002), Epitaxial Growth and Photochemical Annealing of Graded CdS/ZnS Shells on Colloidal CdSe nanorods. J Am Chem Soc 124: 7136-7145.

[44] Coto-Garcia AM, Valledor M, Campo JC, Ferrero FJ, Fernandez-Argüelles MT, CostaFernandez JM, Sanz-Medel A, (2011) Dynamic Analysis of the Photoenhancement Process of Colloidal Quantum Dots with Different Surface Modifications. Nanotechnology, 22: 9. [45] Wu ZY, Zhao YL, Qiu FP, Li YP, Wang SW, Yang BH, Chen L, Sun JH, Wang JY (2009) Forming water-soluble CdSe/ZnS QDs using amphiphilic polymers, stearyl methacrylate/methylacrylate copolymers with different hydrophobic moiety ratios and their optical properties and stability. Colloid Surface A. 350: 121-129.

[46] Karakoti AS, Shukla R, Shanker R, Singh S (2015) Surface functionalization of quantum dots for biological applications. Adv Colloid Interface Sci. 215:28-45.

[47] Aubert T, Soenen SJ, Wassmuth D, Cirillo M, Van Deun R, Braeckmans K, Hens Z (2014) Bright and stable $\mathrm{CdSe} / \mathrm{CdS} @ \mathrm{SiO}(2)$ nanoparticles suitable for long-term cell labelling. ACS Appl Mater Interfaces 6:11714-11723.

[48] Palui G, Aldeek F, Wang WT, Mattoussi H (2015) Strategies for interfacing inorganic nanocrystals with biological systems based on polymer-coating. Chem Soc Rev 44:193-227. [49] Fernandez-Arguelles MT, Costa-Fernandez JM, Pereiro R, Sanz- Medel A (2008) Simple bio-conjugation of polymer-coated quantum dots with antibodies for fluorescencebased immunoassays. Analyst 133:444-447. 
[50] Potapova I, Mruk R, Prehl S, Zentel R, Basche T, Mews A (2003) Semiconductor nanocrystals with multifunctional polymer ligands. J Am Chem Soc 125:320-321.

[51] Mattoussi H, Mauro JM, Goldman ER, Green TM, Anderson GP, Sundar VC, Bawendi MG (2001) Bioconjugation of highly luminescent colloidal CdSe- ZnS quantum dots with an engineered two-domain recombinant protein. Phys Status Solidi B 224: 277-283.

[52] Du Y, Zhong Y, Donga J, Qiana C, Sunc S, Gaod L, Yang D (2019) The effect of PEG functionalization on the in vivo behavior and toxicity of CdTe quantum dots. RSC Adv 9: 12218-12225.

[53] Beloglazova NV, Shmelin PS, Speranskaya ES, Lucas B, Helmbrecht C, Knopp D, Niessner R, De Saeger S, Goryacheva IY (2013) Quantum dot loaded liposomes as fluorescent labels for immunoassay. Anal Chem 85:7197-7204.

[54] Zavari-Nematabad A, Alizadeh-Ghodsi M, Hamishehkar H, Alipour E, PilehvarSoltanahmadi Y, Zarghami N (2017) Development of quantum-dot-encapsulated liposomebased optical nanobiosensor for detection of telomerase activity without target amplification. Anal Bioanal Chem (2017) 409:1301-1310

[55] Kim MW, Jeong HY, Kang SJ, Choi MJ, You YM, Im CS, Lee TS, Song IH, Lee CG, Rhee KJ, Lee YK, Park YS (2017) Cancer-targeted Nucleic Acid Delivery and Quantum Dot Imaging Using EGF Receptor Aptamer-conjugated Lipid Nanoparticles. Sci Rep, 7, Article number: 9474.

[56] Sapsford KE, Algar WR, Berti L, Gemmill KB, Casey BJ, Oh E, Stewart MH, Medintz IL (2013) Functionalizing Nanoparticles with Biological Molecules: Developing Chemistries that Facilitate Nanotechnology. Chem Rev 113:1904-2074.

[57] Boles MA, Ling D, Hyeon T, Talapin DV (2016) The surface science of nanocrystals. Nature Materials 15:141-153.

[58] Howes PD, Chandrawati R, Stevens MM (2014) Colloidal nanoparticles as advanced biological sensors. Science 346:1247390

[59] Hildebrandt N, Spillmann CM, Algar WR, Pons T, Stewart MH, Oh E, Susumu K, Díaz SA, Delehanty JB, Medintz IL (2017) Energy Transfer with Semiconductor Quantum Dot Bioconjugates: A Versatile Platform for Biosensing, Energy Harvesting, and Other Developing Applications. Chem Rev 117:536-711. 
[60] Xia X-R, Monteiro-Riviere NA, Riviere JE (2010) An index for characterization of nanomaterials in biological systems. Nat. Nanotechnol 5:671.

[61] Zhang F, Lees E, Amin F, Rivera-Gil P, Yang F (2011) Polymer-Coated Nanoparticles: A Universal Tool for Biolabelling Experiments. Small 7:3113-3127.

[62] Foubert A, Beloglazova NV, Rajkovic A, Sas B, Madder A, Goryacheva IY, De Saeger S (2016) Bioconjugation of quantum dots: Review \& impact on future application”. Trends Anal Chem 83:31-48.

[63] Karakoti AS, Shukla R, Shanker R, Singh S (2015) Surface functionalization of quantum dots for biological applications. Adv Colloid Interfac 215:28-45.

[64] Pedrero M, Campuzano S, Pingarrón JM (2017) Electrochemical (Bio)sensing of Clinical Markers Using Quantum Dots. Electroanalysis 29:24-37.

[65] Liu W, Li Ch, Ren Y, Sun X, Pan W, Li Y, Wang J, Wang W (2016) Carbon dots: surface engineering and applications. J Mater Chem B 4:5772-5788.

[66] Zhou D, Lin M, Chen Z, Sun H, Zhang H, Sun H, Yang B (2011) Simple Synthesis of Highly Luminescent Water-Soluble CdTe Quantum Dots with Controllable Surface Functionality. Chem Mater 23: 4857-4862.

[67] Heuer-Jungemann A, Feliu N, Bakaimi I, Hamaly M, Alkilany A, Chakraborty I, Masood A, Casula MF, Kostopoulou A, Oh E, Susumu K, Stewart MH, Medintz IL, Stratakis E, Parak WJ, Kanaras AG (2019) The Role of Ligands in the Chemical Synthesis and Applications of Inorganic Nanoparticles. Chem Rev 119:4819-4880.

[68] Sperling RA, Parak WJ (2010) Surface modification, functionalization and bioconjugation of colloidal inorganic nanoparticles. Phil Trans R Soc A 368:1333-1383.

[69] Susumu K, Uyeda HT, Medintz IL, Pons T, Delehanty JB, Mattoussi H (2007) Enhancing the Stability and Biological Functionalities of Quantum Dots via Compact Multifunctional Ligands. J Am Chem Soc 129:13987-13996.

[70] Zhou J, Liu Y, Tang J, Tang W (2017) Surface ligands engineering of semiconductor quantum dots for chemosensory and biological applications. Mater Today 20:360-376.

[71] Erathodiyil N, Ying JY (2011) Functionalization of Inorganic Nanoparticles for Bioimaging Applications. Accounts Chem Res 44:925-935. 
[72] Yu WW, Chang E, Falkner JC, Zhang J, Al-Somali AM, Sayes CM, Johns J, Drezek R, Colvin VL (2007) Forming Biocompatible and Nonaggregated Nanocrystals in Water Using Amphiphilic Polymers. J Am Chem Soc 129:2871-2879.

[73] Wu X, Liu H, Liu J, Haley KN, Treadway JA, Larson JP, Ge NE, Peale F, Bruchez MP (2003) Immunofluorescent labeling of cancer marker Her2 and other cellular targets with semiconductor quantum dots. Nat Biotechnol 21:41-6.

[74] Dubertret B, Skourides P, Norris DJ, Noireaux V, Brivanlou AH, Libchaber a (2002) In Vivo Imaging of Quantum Dots Encapsulated in Phospholipid Micelles. Science 298:1759-1762.

[75] Park J, Lee J, Kwag J, Baek Y, Kim B, Yoon CJ, Bok S, Cho S-H, Kim KH, Ahn GO, Kim S (2015) Quantum Dots in an Amphiphilic Polyethyleneimine Derivative Platform for Cellular Labeling, Targeting, Gene Delivery, and Ratiometric Oxygen Sensing. ACS Nano 9:6511-6521.

[76] Zhou J, Yang Y, Zhang C-Y (2015) Toward Biocompatible Semiconductor Quantum Dots: From Biosynthesis and Bioconjugation to Biomedical Application. Chem Rev 115: 11669-1171.

[77] Qu W, Zuo Q, Li N, Hou Y, Song Z, Gou G, Y J (2017) Design of multifunctional liposome-quantum dot hybrid nanocarriers and their biomedical application. J Drug Target 25:661-672.

[78] Green NM (1963) The Use of [14c]Biotin for Kinetic Studies and for Assay. Biochem. J 89:585-591.

[79] Biju V (2014) Chemical modifications and bioconjugate reactions of nanomaterials for sensing, imaging, drug delivery and therapy. Chem Soc Rev 43:737-962.

[80] Russ Algar W (2017) A Brief Introduction to Traditional Bioconjugate Chemistry. Chap. 1. in Chemoselective and Bioorthogonal Ligation Reactions: Concepts and Applications, Volume 1. Ed.: Algar WR, Dawson PE, Medintz IL. WILEY-VCH Verlag GmbH. \& Co. $\mathrm{KGaA}$. In the other chapters of Volume 1 the reactions mentioned in chapter 1 are dealt in detail.

[81] https://www.thermofisher.com/es/es/home/global/forms/life-science/bioconjugationtechnical-handbook-download.html 
[82] Wagner AM, Knipe JM, Orive G, Peppas NA (2019) Quantum dots in biomedical applications. Acta Biomaterialia 94:44-63.

[83] Lišková M, Voráčová I, Klepárník K, Hezinová V, Přikryl J, Foret F (2011) Conjugation reactions in the preparations of quantum dot-based immunoluminescent probes for analysis of proteins by capillary electrophoresis. Anal Bioanal Chem 400:369-379.

[84] Algar WR, Prasuhn DE, Stewart MH, Jennings TL, Blanco-Canosa JB, Dawson PE, Medintz IL (2011) The Controlled Display of Biomolecules on Nanoparticles: A Challenge Suited to Bioorthogonal Chemistry. Bioconjugate Chem 22:825-858.

[85] Massey M, Algar WR (2017) Nanoparticle Bioconjugates: Materials that Benefit from Chemoselective and Bioorthogonal Ligation Chemistries Chap. 17. in Chemoselective and Bioorthogonal Ligation Reactions: Concepts and Applications, Volume 1. Ed.: Algar WR, Dawson PE, Medintz IL. WILEY-VCH Verlag GmbH \& Co. KGaA.

[86] Row RD, Prescher JA (2018) Constructing New Bioorthogonal Reagents and Reactions. Acc Chem Res 51:1073-1081.

[87] Lv Y, Wu R, Feng K, Li J, Mao Q, Yuan H, Shen H, Chai X, Li LS (2017) Highly sensitive and accurate detection of C-reactive protein by $\mathrm{CdSe} / \mathrm{ZnS}$ quantum dot-based fuorescence-linked immunosorbent assay. J Nanobiotechnol 15:35

[88] Zhang C, Ding C, Zhou G, Xue Q, Xian Y (2017) One-step synthesis of DNA functionalized cadmium-free quantum dots and its application in FRET-based protein sensing . Anal Chim Acta 957:63-69

[89] Long Y, Zhang LF, Zhang Y, Zhang CY (2012) Single quantum dot based nanosensor for renin assay. Anal Chem 84:8846-8852

[90] Sanvicens N, Pascual N, Fernández-Argüelles MT, Adrián J, Costa-Fernández JM, Sánchez-Baeza F, Sanz-Medel A, Marco MP (2011) Quantum dot-based array for sensitive detection of Escherichia coli. Anal Bioanal Chem 399:2755-2762

[91] Wang Y, Gao D, Zhang P, Gong P, Chen C, Gao G, Cai L (2014) A near infrared fluorescence resonance energy transfer based aptamer biosensor for insulin detection in human plasma. Chem Commun 50:811-813

[92] Chen L, Tse WH, Chen Y, McDonaldc MW, Melling J , Zhang J (2017) Nanostructured biosensor for detecting glucose in tear by applying fluorescence resonance energy transfer quenching mechanism. Biosens Bioelectron 91:393-399 
[93] Cayuela A, Soriano ML, Carrillo-Carrión C, Valcárcel M (2016) Semiconductor and carbon-based fluorescent nanodots: the need for consistency. Chem Commun 52:1311-1326 [94] Resch-Genger U, Grabolle M, Cavaliere-Jaricot S, Nitschke R, Nann T(2008) Quantum dots versus organic dyes as fluorescent labels. Nature Methods 5:763-775

[95] Wu S, Liu L, Li G, Jing F, Mao H, Jin Q, Zhai W, Zhang H, Zhao J, Jia C (2016) Multiplexed detection of lung cancer biomarkers based on quantum dots and microbeads. Talanta 156-157:48-54

[96] Zhang W, Hubbard A, Brunhoeber P, Wang Y, Tang L (2013) Automated multiplexing quantum dots in situ hybridization assay for simultaneous detection of ERG and PTEN gene status in prostate cancer. J Mol Diagn 15: 754-764

[97] Han M, Gao X, Su JZ, Nie S (2001) Quantum-dot-tagged microbeads for multiplexed optical coding of biomolecules. Nature Biotechnol 19:631-635

[98] Kim J, Biondi MJ, Feld JJ, Chan WCW (2016) Clinical validation of quantum dot barcode diagnostic technology. ACS Nano 10:4742-4753

[99] Zhao WW, Wang J, Zhu YC, Xu JJ, Chen (2015) Quantum dots: Electrochemiluminescent and photoelectrochemical bioanalysis. Anal Chem 87:9520-9531 [100] Pedrero M, Campuzano S, Pingarrón JM (2017) Electrochemical (bio)sensing of clinical markers using quantum dots. Electroanal 29: 24 - 37

[101] Campuzano S, Yáñez-Sedeño P, Pingarrón JM (2019) Nanoparticles for nucleic-acidbased biosensing: opportunities, challenges, and prospects. Anal Bioanal Chem 411:17911806.

[102] Zheng Y, Wang X, He S, Gao Z, Di Y, Lu K, Li K, Wang J (2019) Aptamer-DNA concatamer-quantum dots based electrochemical biosensing strategy for green and ultrasensitive detection of tumor cells via mercury free anodic stripping voltammetry. Biosens Bioelectron 126:261-268.

[103] Li CC, Hu J, Lu M, Zhang CY (2018) Quantum dot-based electrochemical biosensor for stripping voltammetric detection of telomerase at the single-cell level. Biosens Bioelectron 122:51-57

[104] Liu Y, Zhu L, Kong J, Yang P, Liu B (2013) A quantum dots-based electrochemical assay towards the sensitive detection of tumor cells. Electrochem Comm 33:59-62 
[105] Martín-Yerga D, González-García MB, Costa-García A (2014) Electrochemical immunosensor for anti-tissue transglutaminase antibodies based on the in situ detection of quantum dots. Talanta 130: 598-602

[106] Kong FY, Xu BY, Xu JJ, Chen HY (2013) Simultaneous electrochemical immunoassay using CdS/DNA and PbS/DNA nanochains as labels. Biosens Bioelectron 39:177-182

[107] Christodouleas DC, Kaur B, Chorti P (2018) From point-of-care testing to eHealth diagnostic devices (eDiagnostics). ACS Cent Sci 4: 1600-1616

[108] Tavares AJ, Noor MO, Vannoy CH, Algar WR, Krull UJ (2012) On-chip transduction of nucleic acid hybridization using spatial profiles of immobilized quantum dots and fluorescence resonance energy transfer. Anal Chem 84: 312-319

[109] Noor MO, Tavares AJ, Krull UJ (2013) On-chip multiplexed solid-phase nucleic acid hybridization assay using spatial profiles of immobilized quantum dots and fluorescence resonance energy transfer. Anal Chim Acta 788:148-157

[110] Kim C, Hoffmann G, Searson PC (2017) Integrated magnetic bead-quantum dot immunoassay for malaria detection. ACS Sens 2:766-772

[111] Medina-Sánchez M, Miserere S, Morales-Narváez E, Merkoçi A (2014) On-chip magneto-immunoassay for Alzheimer's biomarker electrochemical detection by using quantum dots as labels. Biosens Bioelectron 54:279-284

[112] Krejcova L, Nejdl L, Merlos-Rodrigo MA, Zurek M, Matousek M, Hynek D, Zitka O, Kopel P, Adam V Kizek, R (2014). 3D printed chip for electrochemical detection of influenza virus labeled with CdS quantum dots. Biosens Bioelectron 54:421-427

[113] Zhou F, Lu M, Wang W, Bian ZP, Zhang JR, Zhu JJ, (2010) Electrochemical Immunosensor for Simultaneous Detection of Dual cardiac markers based on a poly(dimethylsiloxane)-gold nanoparticles composite microfluidic chip: A proof of principle. Clin Chem 56:1701-1707

[114] Gong MM, Sinton D (2017) Turning the page: Advancing paper-based microfluidics for broad diagnostic application. Chem Rev 117: 8447-8480

[115] Roda A, Michelini E, Zangheri M, Di Fusco M, Calabria D, Simoni P (2016) Smartphone-based biosensors: A critical review and perspectives. TrAC Trends Anal Chem 79: $317-325$ 
[116] Bahadır EB, Sezgintürk MK (2016) Lateral flow assays: Principles, designs and labels. TrAC Trends Anal Chem 82:286-306

[117] Martinez AW, Phillips ST, Butte M, Whitesides GM (2007) Patterned paper as a platform for inexpensive, low-volume, portable bioassays. Angew Chem 119: 1340-1342 [118] Urusov AE, Zherdev AV, Dzantiev BB (2019) Towards lateral flow quantitative assays: Detection approaches. Biosensors 9: 89

[119] Fu LM, Wang YN (2018) Detection methods and applications of microfluidic paperbased analytical devices. TrAC Trends Anal Chem 107:196-211

[120] Kokkinos CT, Giokas DL, Economou AS, Petrou PS, Kakabakos SE (2018) Paperbased microfluidic device with integrated sputtered electrodes for stripping voltammetric determination of dna via quantum dot labeling. Anal Chem 90: 1092-1097

[121] Cincotto FH, Fava EL Moraes, FC, Fatibello-Filho O, Faria RC (2019) A new disposable microfluidic electrochemical paper-based device for the simultaneous determination of clinical biomarkers. Talanta 195:62-68

[122] Sapountzi EA, Tragoulias SS, Kalogianni DP,,Ioannou PC, Christopoulos TK, (2015) Lateral flow devices for nucleic acid analysis exploiting quantum dots as reporters. Anal Chim Acta 864: 48-54

[123] Savin M, Mihailescu C-M, Matei I, Stan D, Moldovan CA, Ion M, Baciu I (2018) A quantum dot-based lateral flow immunoassay for the sensitive detection of human heart fatty acid binding protein (hFABP) in human serum. Talanta 178:910-915

[124] Borse V, Srivastava R (2019) Fluorescence lateral flow immunoassay based point-ofcare nanodiagnostics for orthopedic implant-associated infection. Sens Actuat B-Chem 280:24-33

[125] Qina W, Wang K, Xiao K, Hou Y, Lu W, Xu H, Wo Y, Feng S, Cui D (2017) Carcinoembryonic antigen detection with "Handing"-controlled fluorescence spectroscopy using a color matrix for point-of-care applications. Biosens Bioelectron 90:508-515 [126] Liu J, Ji D, Meng H, Zhang L, Wang J, Huang Z, Chen J, Li J, Li Z (2018) A portable fluorescence biosensor for rapid and sensitive glutathionedetection by using quantum dotsbased lateral flow test strip. Sens Actuat B-Chem 262:486-492 
[127] Yan X, Wang K, Lu W, Qin W, Cui D, He J (2016) CdSe/ZnS quantum dot-labeled lateral flow strips for rapid and quantitative detection of gastric cancer carbohydrate antigen 72-4. Nanoscale Res Lett 11:138

[128] Deng X, Wang C, Gao Y, Li J, Wen W, Zhang X, Wang S (2018) Applying strand displacement amplification to quantum dots-based fluorescent lateral flow assay strips for HIV-DNA detection. Biosens Bioelectron 105:211-217

[129] Shah KG, Singh V, Kauffman P, Abe K, Yager P (2018) Mobile phone ratiometric imaging enables highly sensitive fluorescence lateral flow immunoassays without external optical filters. Anal Chem 90:6967-6974.

[130] Wang C, Hou F, Man Y (2015).] Simultaneous quantitative detection of multiple tumor markers with a rapid and sensitive multicolor quantum dots based immunochromatographic test strip. Biosens Bioelectron 68: 156-162

[131] Wu F, Yuan H, Zhou C, Mao M, Liu Q, Shen H, Cen Y, Qin Z, Ma L, Li LS (2016) Multiplexed detection of influenza A virus subtype H5 and $\mathrm{H} 9$ via quantum dot-based immunoassay. Biosens Bioelectron 77: 464-470

[132] Rong Z, Wang Q, Sun N, Jia X, Wang K, Xiao R, Wang S (2019) Smartphone-based fluorescent lateral flow immunoassay platform for highly sensitive point-of-care detection of Zika virus nonstructural protein 1. Anal Chim Acta 1055:140-147

[133] Li X, Li W, Yang Q, Gong X, Guo W, Dong C, Liu J, Xuan L, Chang J (2014) Rapid and quantitative detection of prostate specific antigen with a quantum dot nanobeads-based immunochromatography test strip. Appl Mater Interfaces 6: 6406-6414

[134] Petryayeva E, Algar WR (2015) Single-step bioassays in serum and whole blood with a smartphone, quantum dots and paper in-PDMS chip. Analyst 140: 4037-4045

[135] P Das, UJ Krull (2017) Detection of a cancer biomarker protein on modified cellulose paper by fluorescence using aptamer-linked quantum dots. Analyst 142:3132-3135

[136] Noor MO, Hrovat D, Moazami-Goudarzi M, Espie GS, Krull UJ (2015) Ratiometric fluorescence transduction by hybridization after isothermal amplification for determination of zeptomole quantities of oligonucleotide biomarkers with a paper-based platform and camera-based detection. Anal Chim Acta 885:156-165

[137] Yang M, Liu Y, Jiang X (2019) Barcoded point-of-care bioassays. Chem Soc Rev 48:850-884 
[138] Gao Y, Lam AWY, Chan WCW (2013) Automating quantum dot barcode assays using microfluidics and magnetism for the development of a point-of-care device. ACS Appl Mater.Interfaces 5:2853-2860

[139] Ming K, Kim J, Biondi MJ, Syed A, Chen K, Lam A, Ostrowski M, Rebbapragada A, Feld JJ, Chan WCW (2015) Integrated quantum dot barcodesmartphone optical device for wireless multiplexed diagnosis of infected patients. ACS Nano 9: 3060-3074

[140] Wegner KD, Hildebrandt N (2015) Quantum Dots: Bright and Versatile In Vitro and In Vivo Fluorescence Imaging Biosensors. Chem Soc Rev 44: 4792-4834

[141] Zhao M-X, Zeng E-Z (2015) Application of functional quantum dot nanoparticles as fluorescence probes in cell labeling and tumor diagnostic imaging. Nanoscale Research Letters 10: 171

[142] Liu YS, Sun Y, Vernier PT, Liang CH, Chong SYC, Gundersen MA (2007) pHSensitive Photoluminescence of CdSe/ZnSe/ZnS Quantum Dots in Human Ovarian Cancer Cells. J Phys Chem C 111:2872-2878

[143] Wegner KD, Hildebrandt N (2015) Quantum dots: bright and versatile in vitro and in vivo fluorescence imaging biosensors. Chem Soc Rev 44:4792-4834

[144] Ding H, Yong K-T, Law W-C, Roy I, Hu R, Wu F, Zhao W, Huang K, Erogbogbo F, Bergeya EJ, Prasad PN (2011) Non-invasive tumor detection in small animals using novel functional Pluronic nanomicelles conjugated with anti-mesothelin antibody. Nanoscale 3:1813-1822

[145] Zhang L W, Bäumer W, Monteiro-Riviere N A (2011) Cellular uptake mechanisms and toxicity of quantum dots in dendritic cells. Nanomedicine 6: 777-791.

[146] Xiao Y, Forry S P, Gao X, Holbrook R D, Telford W G, Tona A (2010) Dynamics and mechanisms of quantum dot nanoparticle cellular uptake. J Nanobiotechnol 8, 13.

[147] Igor L, Medintz HTU, Goldman ER, Mattoussi H (2005) Quantum Dot Bioconjugates for Imaging, Labelling and Sensing. Nat Mater 4:435-446.

[148] Rosenthal SJ, Chang JC, Kovtun O, McBride JR, Tomlinson ID (2011) Biocompatible Quantum Dots for Biological Applications. Chem Biol 18:10-24.

[149] Ni X, Castanares M, Mukherjee A, Lupold SE (2011) Nucleic Acid Aptamers: Clinical Applications and Promising New Horizons. Current Medicinal Chem 18: 4206 - 4214. 
[150] Tang J, Huang N, Zhang X, Zhou T, Tan Y, Pi J, Pi L, Cheng S, Zheng H, Cheng Y. (2017) Aptamer-conjugated PEGylated quantum dots targeting epidermal growth factor receptor variant III for fluorescence imaging of glioma. Int J Nanomedicine. 12:3899-3911. [151] McHugh KJ, Jing L, Behrens AM, Jayawardena S, Tang W, Gao M, Langer R, Jaklenec A (2018) Biocompatible Semiconductor Quantum Dots as Cancer Imaging Agents. Adv Mater 1706356

[152] Aswathy RG, Yoshida Y, Maekawa T, Kumar DS (2010) Near-Infrared Quantum Dots for Deep Tissue Imaging. Anal Bioanal Chem 397:1417-1435.

[153] Allen PM, Liu W, Chauhan VP, Lee J, Ting AY, Fukumura D, Jain RK, Bawendi MG (2010) InAs(ZnCdS) Quantum Dots Optimized for Biological Imaging in the Near-Infrared. J Am Chem Soc 132:470-471.

[154] Liu XY, Braun GB, Zhong HZ, Hall DJ, Han WL, Qin MD, Zhao CZ, Wang MN, She ZG, Cao CB, Sailor MJ, Stallcup WB, Ruoslahti E, Sugahara KN (2016) Tumor-Targeted Multimodal Optical Imaging with Versatile Cadmium-Free Quantum Dots. Adv Funct Mater 26:267-276.

[155] Lu C, Chen G, Yu B, Cong H (2018) Recent Advances of Low Biological Toxicity Ag2S QDs for Biomedical Application. Adv Eng Mater 20:1700940.

[156] Wang Z, Ma Y, Yu X, Niu Q, Han Z, Wang H, Li T, Fu D, Achilefu S, Qian Z, Gu Y (2018) Targeting CXCR4-CXCL12 Axis for Visualizing, Predicting, and Inhibiting Breast Cancer Metastasis with Theranostic AMD3100-Ag 2 S Quantum Dot Probe. Adv Funct Mater 28:1800732.

[157] Ortgies DH, García-Villalón AL, Granado M, Amor S, Rodríguez EM, Santos HD, Yao J, Rubio-Retama J, Jaque D (2019) Infrared fluorescence imaging of infarcted hearts with $\mathrm{Ag} 2 \mathrm{~S}$ nanodots. Nano Res 12:749-757.

[158] Hu J, Xie M, Wen C-Y, Zhang Z-L, Xie H-Y, Liu A-A, Chen Y-Y, Zhou S-M, Pang DW (2011) A multicomponent recognition and separation system established via fluorescent, magnetic, dualencoded multifunctional bioprobes. Biomaterials 32:1177-1184

[159] Xu H-L, Yang JJ, ZhuGe DL, Lin MT, Zhu QY, Jin BH, Tong MQ, Shen B-X, Xiao J, Zhao Y-Z (2018) Glioma-Targeted Delivery of a Theranostic Liposome Integrated with Quantum Dots, Superparamagnetic Iron Oxide, and Cilengitide for Dual-Imaging Guiding Cancer Surgery. Adv Healthcare Mater 7:1701130. 
[160] Deng Y, Xu A, Yu Y, Fu C, Liang G (2018) Biomedical applications of fluorescent and magnetic resonance imaging dual-modality probe. ChemBioChem 20:499

[161] Yang Y, Lin L, Jing L, Yue X, Dai Z (2017) CuInS2/ZnS Quantum Dots Conjugating Gd(III) Chelates for Near-Infrared Fluorescence and Magnetic Resonance Bimodal Imaging. ACS Appl Mater Interfaces 9:23450-23457

[162] Estelrich J, Sánchez-Martín MJ, Busquets MA (2015). Nanoparticles in magnetic resonance imaging: from simple to dual contrast agents. International journal of nanomedicine 10: 1727-1741.

[163] Garcia-Cortes M, Ruiz Encinar J, Costa-Fernandez JM, Sanz-Medel A (2016) Highly sensitive nanoparticle-based immunoassays with elemental detection: Application to Prostate-Specific Antigen quantification. Biosens Bioelect 85: 128-134.

[164] Chong Y, Ma Y, Shen H, Tu X, Zhou X, Xu J, Dai J, Fan S, Zhang Z (2014) The in vitro and in vivo toxicity of graphene quantum dots. Biomaterials 35:5041-5048 